\title{
A moving boundary problem motivated by electric breakdown, I: Spectrum of linear perturbations
}

\author{
S. Tanveer ${ }^{\mathrm{a}}$, L. Schäfer ${ }^{\mathrm{b}}$, F. Brau ${ }^{\mathrm{c}}, \mathrm{U}$. Ebert ${ }^{\mathrm{c}, *}$ \\ a Department of Mathematics, Ohio State University, 231 W 18th Ave, Columbus 43210, USA \\ ${ }^{\mathrm{b}}$ Fachbereich Physik, Universität Duisburg-Essen, Lotharstr. 1, 47048 Duisburg, Germany

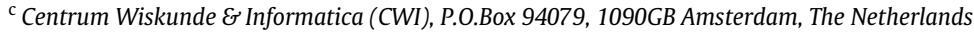

\section{A R T I C L E I N F O}

\section{Article history:}

Received 1 September 2008

Received in revised form

17 February 2009

Accepted 20 February 2009

Available online 9 March 2009

Communicated by B. Sandstede

\section{PACS:}

47.54.-r

Keywords:

Moving boundary

Kinetic undercooling regularization

Linear stability analysis

Laplacian instability

Electric breakdown

\begin{abstract}
A B S T R A C T
An interfacial approximation of the streamer stage in the evolution of sparks and lightning can be written as a Laplacian growth model regularized by a 'kinetic undercooling' boundary condition. We study the linear stability of uniformly translating circles that solve the problem in two dimensions. In a space of smooth perturbations of the circular shape, the stability operator is found to have a pure point spectrum. Except for the eigenvalue $\lambda_{0}=0$ for infinitesimal translations, all eigenvalues are shown to have negative real part. Therefore perturbations decay exponentially in time. We calculate the spectrum through a combination of asymptotic and series evaluation. In the limit of vanishing regularization parameter, all eigenvalues are found to approach zero in a singular fashion, and this asymptotic behavior is worked out in detail. A consideration of the eigenfunctions indicates that a strong intermediate growth may occur for generic initial perturbations. Both the linear and the nonlinear initial value problem are considered in a second paper.
\end{abstract}

(C) 2009 Elsevier B.V. All rights reserved.

\section{Introduction}

The motion of interfaces in a Laplacian field is of general interest and has been a subject of intense study over many years (see for instance the reviews $[1,2])$. Such problems arise in many physical contexts, such as viscous fingering in multi-phase fluid flow [3-16], dendritic crystal growth in the quasi-steady small Peclet number limit [17-20], void electromigration [21-25] and a host of other phenomena such as the growth of biological systems like bacterial colonies or corals [26].

More recently, a similar mathematical problem has been discovered in the study of 'streamers' [27-34] which occur during the initial stage of electric breakdown and play an important role both in the natural phenomena of sparks and lightning as well as in numerous technical applications [33]. Streamers are weakly ionized bodies growing into some nonionized medium due to an externally applied electric field. This field is so strong that the drifting electrons very efficiently create additional electron ion pairs by impact ionization, and the nonlinear coupling between ionized body and field further increases this effect.

\footnotetext{
* Corresponding author.

E-mail address: ebert@cwi.nl (U. Ebert).
}

Models for negative streamers in simple gases like nitrogen or argon are based on a set of partial differential equations for the densities of electrons and of positive ions coupled to the electric field [28-33]. Analysis and numerical solutions of these equations reveal that in the front part of the streamer, a thin surface charge layer develops where the electron density strongly exceeds the ion density. Therefore the electric field $\mathbf{E}$ varies strongly when crossing this layer. Right before the layer, it is enhanced, but in the interior of the streamer, it is screened to such a low level that impact ionization is suppressed and the electron current transporting charge from the interior to the surface charge layer is small. Consequently we may take the interior as being essentially passive, and the growth of the streamer is governed by the surface charge layer which is driven by the strong local field.

If the external field is very strong, the thickness $\ell$ of the surface charge layer can become small compared to the typical diameter $2 R$ of the streamer [34]. This suggests modeling this layer as an interface separating the ionized interior from the nonionized exterior region. In this model the variation of the potential $\varphi$ across the surface charge layer is replaced by a discontinuity on the interface. Since the interior is considered passive, only the limiting value $\varphi^{+}$reached by approaching the interface from the outside is relevant for the dynamical evolution, and analysis of results of the PDE-model suggests [34-36] that with an appropriate gauge, $\varphi^{+}$is coupled to the limiting value $\mathbf{E}^{+}$of the electric field by the 
boundary condition

$\varphi^{+}=-\ell \mathbf{n} \cdot \mathbf{E}^{+}$.

Here $\mathbf{n}$ is the outward normal on the interface, $\ell$ is the regularization length corresponding to the interface thickness, and $\mathbf{E}^{+}=-\nabla \varphi^{+}$, where the ${ }^{+}$again indicates the limit of approaching the interface from the outside. In the context of dendritic crystal growth, this boundary condition is equivalent to including the kinetic undercooling effect ${ }^{1}$ while excluding the usual Gibbs-Thompson surface energy correction to the melting temperature.

As the motion of the interface is caused by the drift of the electrons in the local electric field $\mathbf{v}=-\mathbf{E}$, the interface moves with normal velocity

$v_{n}=-\mathbf{n} \cdot \mathbf{E}^{+}$,

and outside the streamer the potential obeys the Laplace equation

$\Delta \varphi=0$.

We discuss the problem defined by Eqs. (1)-(3) in infinite two-dimensional space, with the electric field becoming constant, $\mathbf{E}=-\nabla \phi \rightarrow \mathbf{E}_{\infty}$, far from the streamer; such a condition is realized frequently in atmospheric discharges, e.g., inside thunderclouds. This far-field condition on $\nabla \phi$ is different from the usual source/sink condition in viscous fingering in the absence of side-walls, or undercooling specification in the quasi-steady low Peclet number crystal growth problem which have been extensively studied. However, moving bubbles and fingers in a long Hele-Shaw channel are indeed subjected to this type of far-field condition. (For the discussion of the streamer problem equivalent to the Saffman-Taylor finger, we refer to [38].) Further, in the crystal growth or directional solidification problem, a systematic inner-outer analysis for small Peclet number $[17,20]$ shows that this is an appropriate condition at $\infty$ for the "inner" problem.

A simple steady solution to the streamer equations given above is a circle translating with constant velocity determined by $\mathbf{E}_{\infty}$ $[35,36]$. Though such circles differ from proper streamers, which are growing channels of ionized matter [33,38-40], their front half closely resembles the head of the streamer where the growth takes place. It is therefore a question of physical interest whether or not translating circular solutions are stable to small perturbations and this is the subject of the present investigation.

The relevance of this analysis for more realistic streamer shapes is supported by results found in another physical context. Steadily translating circles also arise in viscous fingering in a Hele-Shaw cell when surface tension is included (instead of kinetic undercooling) in the limit when the bubble is small compared to the cell-dimensions [3]. The linear stability of these bubbles, including larger non-circular steadily translating bubbles, has been studied before both for one and two fluids $[10,14]$ and the results largely mimic those obtained for a finger, though the latter calculations are mathematically much more involved.

It has been known for a while that in the absence of any regularization, such as surface tension or kinetic undercooling, the initial value problem in a Laplacian field is ill-posed [9] in any norm that is physically relevant to describing interfacial features. This is reflected in the instability of any steady shape, when the growth rates increase with the wave numbers of the disturbances. Ill-posedness makes idealized model predictions sometimes physically irrelevant (see [15] for a thorough discussion) and regularization becomes essential.

\footnotetext{
1 Under most natural conditions of crystal growth, kinetic undercooling is important in a limit when the Peclet number is not small enough to justify a Laplacian field approximation; nonetheless, there have been some studies of steady Laplacian crystal growth with kinetic undercooling effects only [37].
}

Considering a planar front, one finds that regularization does not remove the instability against fluctuations of small wave number. However, for large wave numbers, linear stability analysis exhibits a basic difference between surface tension and kinetic undercooling. All large wave number components of a disturbance decay with surface tension regularization, while for kinetic undercooling the growth rate saturates to a constant that scales as $\ell^{-1}$ [16]; for streamers, such a saturating dispersion relation is derived and discussed in $[41,42]$.

For curved fronts, one can pose the question: how does curvature stabilize, if at all, a disturbance whose wavenumber is in the unstable regime for a flat interface, either with surface tension or with kinetic undercooling regularization? With surface tension regularization, some answers are available in the existing literature. Arguments have been presented $[4,6]$ that suggest that a localized wave packet with wave numbers in the unstable regime ${ }^{2}$ advects along the front as it grows; once it reaches the side of the front where the local normal velocity is zero, the disturbance stops growing. If the steady shape is closed, as it is for a circle, the continued advection of the wave-packet towards the receding parts of the interface will cause the disturbance to decay eventually. ${ }^{3}$ If regularization is small, there is a large transient growth. Unless the disturbance amplitude is smaller than a threshold that shrinks to zero with regularization, the transient exponential growth causes the interface to enter a nonlinear regime that can destabilize the steady front, even when it is predicted to be linearly stable. Analysis of approximate equations, supported by numerical calculations of the full equations support the above scenario. Similar stabilization should occur for the kinetic undercooling boundary condition as well, though we are not aware of any explicit study affirming this expectation.

Note, however, that stabilization of localized wave packets does not rule out instability to long ranged disturbances. A formal asympotic study for small nonzero surface tension [12,14] as well as numerical studies [7] reveal that surface tension stabilizes precisely one branch of steady solutions for fingers and bubbles in a Hele-Shaw cell. Similar results follow for a needle crystal [18] though in the latter case, convective instability of wave packets caused by significant normal speed along the parabolic front is believed to cause dendritic structures [1]. These conclusions have been challenged at times by alternate scenarios (see for instance [19]) that are based on formal calculations, but with different implicit assumptions. Such controversy affirms the need for more rigorous mathematical studies of the stability problem, even if it is for relatively simple shapes such as the circle in the present study.

For the kinetic undercooling boundary condition, relying merely on a numerical study to understand the long time behavior is fraught with difficulties. One finds a collapsing spatial scale for large time at the rear of the circle. Analytically, this is found for $\epsilon=\ell / R=1$ in $[35,36] .{ }^{4}$ As will be argued in the present and the companion paper, the occurrence of this collapsing scale is a general feature for any $\epsilon>0$. This means that as $t \rightarrow \infty$, one must resolve progressively finer scales near the back of the bubble. Further, calculations for small $\epsilon$ require resolving a large number of transiently growing modes. All this underscores the need of some progress on the analytical side.

\footnotetext{
2 Localized disturbances refer to those with wavelengths far smaller than the typical radius of curvature of the steady shape. These can be unstable only if the regularization parameter is sufficiently small.

3 Surface tension causes localized disturbances to decay as they advect to the sides even when an interface is not closed but becomes parallel to the direction of motion as is the case for a finger in a Hele-Shaw cell. However, no decay is expected for kinetic regularization. This is where a closed interface is different.

4 We recall that $R$ is a measure of the size of the streamer. The precise definition is given in Eq. (5)
} 
The present paper, which is part I of a two-paper sequence, is devoted to the spectral properties of the linear stability operator, associated with infinitesimal perturbations of a circle. In part II [43], we will consider the initial value problem, presenting analytical and numerical results on the evolution of both infinitesimal and finite perturbations.

The present paper is organized as follows. In Section 2 we reformulate the problem defined by Eqs. (1)-(3) by standard conformal mapping, and we present the PDE governing the time evolution of infinitesimal perturbations of the circle. This material has been presented before in $[35,36]$, where also the general solution of the PDE in the case $\epsilon=1$ has been discussed in detail. The explicit solution found for $\epsilon=1$ shows that outside any fixed neighborhood of the rear of the bubble, the long-term behavior of infinitesimal perturbations is described by $\sum_{n=0}^{\infty} \mathrm{e}^{\lambda_{n} t} \beta_{\lambda_{n}}$, where $\lambda_{n}$ is the $n$th eigenvalue (ordered according to absolute value) of the linear stability operator and $\beta_{\lambda_{n}}$ is the corresponding eigenfunction.

We then study this eigenvalue value problem for arbitrary $\epsilon>0$. We show in Section 3 that the linear stability operator, defined in an appropriate space of analytic functions, has a pure point spectrum. In Section 4 it is proven that there are no discrete eigenvalues with non-negative real part, except $\lambda=0$ that corresponds to the trivial translation mode. A set of discrete, purely negative eigenvalues is calculated in Section 5 as a function of $\epsilon$; they smoothly extend the results found previously for $\epsilon=1$. The results suggests that as $\epsilon \rightarrow 0$, the spectrum degenerates to the trivial translation mode and this limit is discussed in detail in Section 6. Section 7 contains a discussion of the eigenfunctions belonging to these eigenvalues, and Section 8 contains the conclusions. Some part of our analysis exploits general results on the asymptotic behavior of the coefficients of Taylor expansions. These results are presented in an Appendix.

\section{Reformulation by conformal mapping}

In this section, we collect results and notations from $[35,36]$ that will be used in later sections.

\subsection{Problem formulation and rescaling}

We consider a compact ionized domain $\mathscr{D}$ in the $(x, y)$-plane. We assume that the net charge on the domain vanishes (i.e., it contains the same number of electrons and positive ions). The domain moves in an external field that far from the domain asymptotically approaches

$\mathbf{E}_{\infty}=-\left|\mathbf{E}_{\infty}\right| \hat{\mathbf{x}}$.

Here $\hat{\mathbf{x}}$ is the unit vector in $x$-direction, and $\left|\mathbf{E}_{\infty}\right|$ sets the scale of $\mathbf{E}$ and thus of the potential $\varphi$. As length scale we take

$R=\sqrt{\frac{|\mathscr{D}|}{\pi}}$.

where $|\mathscr{D}|$ is the area of $\mathscr{D}$, which is known to be conserved. This follows from the charge neutrality of the streamer since

$0=\int_{\mathscr{D}} \mathrm{d} x \mathrm{~d} y \nabla \cdot \mathbf{E}=\int_{\partial \mathcal{D}} \mathrm{d} s \mathbf{n}(s) \cdot \mathbf{E}=-\int_{\partial \mathcal{D}} \mathrm{d} s v_{n}$,

where in the last step we inserted Eq. (2) for the normal velocity of the boundary. Since

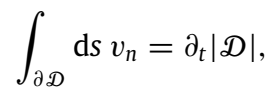

the area is conserved, irrespective of the precise charge distribution in the interior. ${ }^{5}$ Also introducing the time scale $R /\left|E_{\infty}\right|$, we

\footnotetext{
5 We remark that the argument is straight forward to generalize to three spatial dimensions. Therefore the volume of a charge neutral object with surface velocity $\mathbf{v} \propto \mathbf{E}^{+}$in three spatial dimensions is conserved as well.
}

rescale the basic equations to the dimensionless form

$\Delta \varphi=0, \quad(x, y) \notin \mathscr{D}$

$v_{n}=\mathbf{n} \cdot(\nabla \varphi)^{+}$

$\varphi^{+}=\epsilon \mathbf{n} \cdot(\nabla \varphi)^{+}$.

The only remaining parameter in the rescaled problem is

$\epsilon=\ell / R$.

The boundary condition at infinity after rescaling takes the form

$\varphi \rightarrow x+$ const for $\sqrt{x^{2}+y^{2}} \rightarrow \infty$.

\subsection{Conformal mapping}

We now identify the physical $(x, y)$-plane with the closed complex plane $z=x+\mathrm{i} y$, and we introduce a conformal map $f(\omega, t)$ that maps the unit disk $U_{\omega}$ in the $\omega$-plane to the complement of $\mathscr{D}$ in the $z$-plane, with $\omega=0$ being mapped on $z=\infty$,

$z=f(\omega, t)=\frac{a_{-1}(t)}{\omega}+\hat{f}(\omega, t), \quad a_{-1}(t)>0$.

We further define a complex potential $\Phi(\omega, t)$ obeying

$\operatorname{Re}[\Phi(\omega, t)]=\varphi(f(\omega, t)) \quad$ for $\omega \in \mathcal{U}_{\omega}$.

The boundary condition (12) and the Laplace Eq. (8) enforce the form

$\Phi(\omega, t)=\frac{a_{-1}(t)}{\omega}+\hat{\Phi}(\omega, t)$

with $\hat{\Phi}$ being holomorphic for $\omega \in U_{\omega}$.

The two boundary conditions (9) and (10) take the form

$\operatorname{Re}\left[\frac{\partial_{t} f}{\omega \partial_{\omega} f}\right]=\operatorname{Re}\left[\frac{\omega \partial_{\omega} \Phi}{\left|\partial_{\omega} f\right|^{2}}\right]$ for $\omega \in \partial u_{\omega}$,

$\left|\partial_{\omega} f\right| \operatorname{Re}[\Phi]=-\epsilon \operatorname{Re}\left[\omega \partial_{\omega} \Phi\right]$ for $\omega \in \partial u_{\omega}$,

which completes the reformulation of the moving boundary problem (8)-(12) by conformal mapping.

We will restrict the analysis here to initial conditions $\hat{f}(\omega, 0)$ holomorphic in some domain $u_{0}^{\prime} \supset u_{\omega}$. In part II [43] of this paper sequence, we will give evidence that analyticity on $\bar{U}_{\omega}$ is preserved in time, though the distance of the domain of analyticity $U_{t}^{\prime}$ to $\partial u_{\omega}$ shrinks with time. The streamer boundary $\partial \mathcal{D}$, which is the image of boundary $\partial u_{\omega}$ under $f(\omega, t)$, will turn out to be analytic and therefore smooth. Similar analytic representations exist for the entire class of 2-D Laplacian growth, with details depending on the type of boundary condition, geometry and asymptotic conditions at infinity. For the classic viscous fingering problem, PolubarinovaKochina [44] and Galin [45] use a representation that coincides with the one given above in the unregularized case $\epsilon=0$.

\subsection{Linear perturbation of moving circles}

It is easily seen that Eqs. (16) and (17) allow for the simple solution

$f^{(0)}(\omega, t)=\frac{1}{\omega}+\frac{2 t}{1+\epsilon}$,

$\Phi^{(0)}(\omega, t)=\frac{1}{\omega}-\frac{1-\epsilon}{1+\epsilon} \omega$,

which in physical space describes circles of radius 1 moving with constant velocity $2 /(1+\epsilon)$ in $x$ direction. (We recall that the radius was scaled to unity in Section 2.1.) We note that relaxing 
the analyticity conditions on $f(\omega, t)$ on $|\omega|=1$, one can obtain another set of uniformly translating solutions, as recently discovered [46]. The present paper is restricted to perturbations of the steady circle that retain the imposed analyticity of the streamer shapes, and hence analyticity of $f$ (as well as $\Phi$ ) on $|\omega|=1$.

As the area is conserved (as shown in Section 2.1), the residue $a_{-1}=1$ does not change to linear order in the perturbation. We therefore can use the ansatz

$f(\omega, t)=f^{(0)}(\omega, t)+\eta \beta(\omega, t)$,

$\Phi(\omega, t)=\Phi^{(0)}(\omega, t)+\eta \frac{2}{1+\epsilon} \chi(\omega, t)$,

where $\eta$ is a small parameter, and $\beta(\omega, t), \chi(\omega, t)$ are holomorphic in $U_{\omega}$. A first order expansion of Eqs. (16) and (17) in $\eta$ yields the following boundary conditions for the analytic functions $\beta(\omega, t)$ and $\chi(\omega, t)$ on $|\omega|=1$ :

$\operatorname{Re}\left[\omega \partial_{\tau} \beta-\omega \partial_{\omega} \beta\right]=\operatorname{Re}\left[-\omega \partial_{\omega} \chi\right]$,

$\frac{\epsilon}{2} \operatorname{Re}\left[\left(\omega+\frac{1}{\omega}\right) \omega^{2} \partial_{\omega} \beta\right]=\operatorname{Re}\left[\epsilon \omega \partial_{\omega} \chi+\chi\right]$,

where we rescaled time as

$\tau=\frac{2}{1+\epsilon} t$.

Since the left and right sides of each of the two equations in (20) are real parts of analytic functions and each is assumed a priori continuous up to the boundary, they can differ everywhere in $\omega$ by at most an imaginary constant. Evaluation at $\omega=0$ shows this constant to be zero for the first of the two equations. Elimination of $\chi$ results in the linear PDE:

$\mathcal{L}_{\epsilon} \beta=0$

with the operator

$\mathcal{L}_{\epsilon}=\frac{\epsilon}{2} \partial_{\omega}\left(\omega^{2}-1\right) \omega \partial_{\omega}+\epsilon \partial_{\omega} \omega \partial_{\tau}+\partial_{\tau}-\partial_{\omega}$.

We note that $\mathcal{L}_{\epsilon}$ is of similar structure as the operator resulting from a linear stability analysis of translating circles in the context of void electromigration $[22,24]$. The main difference here is the occurrence of the mixed derivative $\partial_{\omega} \omega \partial_{\tau}$.

\subsection{Formulation of the eigenvalue problem}

To motivate our formulation of the eigenvalue problem, we note some results on the temporal evolution of infinitesimal perturbations. In [36], the equation $\mathcal{L}_{\epsilon} \beta=0$ was solved as an initial value problem for the special value $\epsilon=1$. It was found that any initial perturbation $\beta(\omega, 0)$ holomorphic in $U^{\prime} \supset U_{\omega}$ for $\tau \rightarrow \infty$ is exponentially convergent to some constant. This results from the expansion

$\beta(\omega, \tau)=\sum_{n=0}^{\infty} g_{n} \beta_{\lambda_{n}}^{(1)}(\omega) \mathrm{e}^{\lambda_{n} \tau}$,

with

$\lambda_{n}=-n, \quad n \in \mathbb{N}_{0}$, for $\epsilon=1$.

The coefficients $g_{n}$ and the eigenfunctions ${ }^{6}$

$\beta_{\lambda_{n}}^{(1)}(\omega)=\int_{0}^{\omega} \frac{x \mathrm{~d} x}{\omega^{2}}\left(\frac{x-1}{x+1}\right)^{-\lambda_{n}}$

\footnotetext{
6 Note that the exponent $-\lambda_{n}$ in (26) is correct while $+\lambda_{n}$ in Eq. (4.20) in [36] is a typo.
}

are determined by an expansion of $\left(2+\omega \partial_{\omega}\right) \beta(\omega, 0)$ in powers of $(1-\omega) /(1+\omega)$. For $n>0$ the eigenfunctions (26) are singular at $\omega=-1$, though $\beta(\omega, \tau)$ is not. The expansion (24) is convergent in a domain $\mathscr{D}_{\tau}$ expanding in time that eventually includes every point in $\bar{u}_{\omega} \backslash\{-1\}$. For large $\tau$ the region where the expansion is invalid, shrinks to $\omega=-1$ exponentially. This region is measured by the new scale $\eta_{1}(\omega, \tau)=(1+\omega) \mathrm{e}^{\tau}$, and the expansion (24) is valid if $\eta_{1}$ is large. For $\eta_{1} \leq O(1)$ the perturbation for $\tau \rightarrow \infty$ behaves as $\beta(\omega, \tau) \rightarrow F_{0}\left(\eta_{1}\right)+O\left(\mathrm{e}^{-\tau}\right)$ where $F_{0}$ is some analytic function of its argument, depending on $\beta(\omega, 0)$.

For an analytic initial condition on $\mathcal{U}^{\prime}$, with a lone branch point singularity $\omega_{s}$ in $|\omega|>1$ not on the positive real axis, the emergence of this new scale near $\omega=-1$ can be related to the approach of this complex singularity towards -1 exponentially in $\tau$ for large $\tau$. Asymptotic arguments that will be presented in part II [43] suggest that this behavior is generic for all $\epsilon>0$. The analysis is based on the linear the evolution equations for $b_{k}$, where

$\beta(\omega, \tau)=\sum_{k=0}^{\infty} b_{k}(\tau) \omega^{k}$.

For $k \gg \mathrm{e}^{\tau}$, we find the asymptotic relation

$b_{k} \sim(-1)^{k} k^{-\alpha} h(\tau) \exp [-k f(\tau)]$,

where

$f(\tau)=\log \left[\frac{1+C \mathrm{e}^{-\tau}}{1-C \mathrm{e}^{-\tau}}\right], \quad$ with $C=\frac{\omega_{s}+1}{\omega_{s}-1}$.

For $\omega_{s} \notin(1, \infty), f(\tau)$ stays finite and approaches 0 exponentially in $\tau$ for large $\tau$. If $\omega_{s} \in(1, \infty), f(\tau)$ increases monotonically to $\infty$ for $\tau \in\left(0, \tau_{c}\right)$ where $\mathrm{e}^{-\tau_{c}}=1 / C$. For $\tau>\tau_{c}, f(\tau)$ decreases monotonically and approaches 0 exponentially in $\tau$ as $\tau \rightarrow \infty$. In either case, from the known relation between Taylor series coefficients and the location of the closest singularity of an analytic function (see the Appendix), it follows that $f(\tau) \sim \mathrm{e}^{-\tau}$ as $\tau \rightarrow \infty$ implies that $\beta$ has a singularity approaching $\omega=-1$ exponentially in $\tau$ for large $\tau$. This feature is retained for any other isolated initial singularities as well, though $k^{-\alpha}$ is replaced by a more complicated dependence in $k$. Since the problem is linear, the evolution of a distribution of initial singularities can be understood from the linear superposition principle.

This suggests that for any $\epsilon>0$, as for $\epsilon=1, \beta(\omega, \tau)$ has a collapsing scale $(1+\omega) \mathrm{e}^{\tau}$, and an expansion of the type (24) cannot be valid in this neighborhood of $\omega=-1$.

Thus, in seeking an eigenfunction by substituting

$\beta(\omega, \tau)=\beta_{\lambda}^{(\epsilon)}(\omega) \mathrm{e}^{\lambda \tau}$,

into (22) and (23), it is appropriate to allow $\beta_{\lambda}^{(\epsilon)}$ to be singular at $\omega=-1$. Indeed, substituting the form (27) reduces Eqs. (22) and (23) to the eigenvalue problem

$$
\begin{aligned}
L(\epsilon, \lambda) \beta_{\lambda}^{(\epsilon)}(\omega)=0, & \\
L(\epsilon, \lambda)= & \frac{\epsilon\left(\omega^{2}-1\right) \omega}{2} \partial_{\omega}^{2}+\left(\frac{\epsilon\left(3 \omega^{2}-1\right)}{2}-1\right) \partial_{\omega} \\
& +\lambda\left(1+\epsilon+\epsilon \omega \partial_{\omega}\right) .
\end{aligned}
$$

Evidently this ODE has three regular singular points, namely $\omega=0$ and $\omega= \pm 1$. The independent solutions at these points for $\epsilon>0$ are in leading order

$\beta_{\lambda}^{(\epsilon)}(\omega) \sim\left\{\begin{array}{c}\omega^{0} \\ \omega^{-2 / \epsilon}\end{array}\right.$ for $\omega \rightarrow 0$,

$\beta_{\lambda}^{(\epsilon)}(\omega) \sim\left\{\begin{array}{c}(1 \mp \omega)^{0} \\ (1 \mp \omega)^{1 / \epsilon \mp \lambda}\end{array}\right.$ for $\omega \rightarrow \pm 1$ 
We require the eigenfunctions $\beta_{\lambda}^{\epsilon}$ to be solutions of (28) that are analytic in $\omega=0$ and $\omega=1$. This is also the natural choice from a physical point of view since it is the right half of the circle, $\operatorname{Re}[\omega]>0$, that corresponds to the physically interesting tip of the streamer. In general, eigenfunctions cannot be expected to be regular at all three points. Starting with a function regular at $\omega=0$, we cannot generally require regularity at both points $\omega= \pm 1$ by adjusting the single parameter $\lambda$. As shown in Section 4.3, the only eigenfunction regular at all three points is the trivial translation mode

$\lambda_{0}=0, \quad \beta_{0}^{(\epsilon)}(\omega)=$ const.

As noted above, an operator similar to $\mathcal{L}_{\epsilon}(23)$ occurs in the problem of void electromigration, see section 4.1.3 in [24]. Again an eigenvalue analysis would yield a second order linear operator with three singular points at $\omega=0$ and \pm 1 and therefore the eigenmodes in general cannot be regular at all three singular points. It is interesting to note that the authors [24] conclude that their problem is unstable because the initial value problem for large time is singular at $\omega=-1$. In the current problem, the solution [43] of the initial value problem is not singular at $\omega=-1$; the singularity of the eigenfunctions does not reflect the true behavior of solution since, as has been pointed out earlier, there is an anomalous contracting scale $\mathrm{e}^{\tau}(1+\omega)$ near the back of the bubble. Whether or not there is an analogous contracting scale for the void electromigration problem [24] remains an interesting question. This anomalous scale shows up when the limiting processes $\lim _{\omega \rightarrow-1}$ and $\lim _{\tau \rightarrow+\infty}$ do not commute for the solution of the initial value problem.

\section{Discreteness of the spectrum}

We define $\lambda$ to be in the spectrum, if the linear operator $L(\epsilon, \lambda)$ does not have a bounded inverse in the class of functions $f$ that are analytic in an arbitrary compact connected set $\mathcal{V} \subset U^{\prime} \backslash\{-1\}$ that contains the whole line $[0,1]$ in its interior. $\lambda$ is in the discrete spectrum if (28) has a nonzero solution $\beta_{\lambda}^{(\epsilon)}(\omega)$ that is analytic in any such domain $\mathcal{V}$. We now argue that if $\lambda$ is not in the discrete spectrum, then $L(\epsilon, \lambda)$ has a bounded inverse, i.e. there is only a discrete spectrum in this problem.

To determine $L^{-1}$, we solve the equation

$L(\epsilon, \lambda) g=h$

for a given $h$ analytic in $\mathcal{V}$, imposing the condition that also $g$ is analytic in $\mathcal{V}$. The solutions of the homogeneous equation $L(\epsilon, \lambda) f=0$ that are regular at $\omega=0$ or $\omega=1$ will be denoted by $f_{1}(\omega)$ or $f_{2}(\omega)$, respectively. It follows from Eqs. (30) and (31) that these functions are determined uniquely up to a multiplicative constant. In the exceptional case where both independent solutions are regular at $\omega=1, \lambda$ belongs to the discrete spectrum, see Section 5. A standard calculation shows that Eq. (33) is solved by

$g(\omega)=\frac{1}{C(\epsilon, \lambda)} \int_{0}^{\omega} \mathrm{d} \omega^{\prime} G\left(\omega, \omega^{\prime}\right) h\left(\omega^{\prime}\right)+a_{1}[h] f_{1}(\omega)$,

where

$$
\begin{aligned}
G\left(\omega, \omega^{\prime}\right)= & \frac{\omega^{\prime 2 / \epsilon}}{\left(1-\omega^{\prime}\right)^{1 / \epsilon-\lambda}\left(1+\omega^{\prime}\right)^{1 / \epsilon+\lambda}} \\
& \times\left[f_{2}(\omega) f_{1}\left(\omega^{\prime}\right)-f_{1}(\omega) f_{2}\left(\omega^{\prime}\right)\right],
\end{aligned}
$$

and the coefficient $a_{1}[h]$ is a functional of $h\left(\omega^{\prime}\right) . C(\lambda, \epsilon)$ does not vanish since otherwise the Wronskian $f_{1} \partial_{\omega} f_{2}-f_{2} \partial_{\omega} f_{1}$ vanishes identically and $\lambda$ is part of the discrete spectrum. It is easily seen that Eqs. (34) and (35) render $g(\omega)$ analytic in $\omega=0$, and this condition eliminates any contribution of the form $a_{2}[h] f_{2}(\omega)$.
Analyticity at $\omega=1$ is enforced by a proper choice of $a_{1}[h]$. To make the analysis explicit, in addition to $f_{2}(\omega)$, we introduce another solution to $L[\epsilon, \lambda] f=0$ by requiring

$f_{3}(\omega)=(1-\omega)^{1 / \epsilon-\lambda} \hat{f}_{3}(\omega)$,

where $\hat{f}_{3}(\omega)$ is analytic at $\omega=1$. Using this form of $f_{3}(\omega)$, we exclude the case $\frac{1}{\epsilon}-\lambda \in \mathbb{Z}^{+}$, that will be discussed later. Writing $f_{1}(\omega)$ as

$f_{1}(\omega)=c_{2} f_{2}(\omega)+c_{3} f_{3}(\omega)$,

we find that $G\left(\omega, \omega^{\prime}\right)$ from Eq. (35) takes the form

$$
\begin{aligned}
G\left(\omega, \omega^{\prime}\right)= & \frac{c_{3} \omega^{\prime 2 / \epsilon}}{\left(1+\omega^{\prime}\right)^{1 / \epsilon+\lambda}} \\
& \times\left[f_{2}(\omega) \hat{f}_{3}\left(\omega^{\prime}\right)-\left(\frac{1-\omega^{\prime}}{1-\omega}\right)^{\lambda-1 / \epsilon} \hat{f}_{3}(\omega) f_{2}\left(\omega^{\prime}\right)\right] .
\end{aligned}
$$

Evidently the first part in the square brackets for $\omega \rightarrow 1$ yields a regular contribution to $g(\omega)$ from Eq. (34). The contribution to $\int G h$ that is singular in $\omega=1$ has the form

$-c_{3} f_{3}(\omega) \int_{0}^{\omega} \mathrm{d} \omega^{\prime}\left(1-\omega^{\prime}\right)^{\lambda-1 / \epsilon} H\left(\omega^{\prime}\right)$,

where

$H\left(\omega^{\prime}\right)=\frac{\omega^{\prime 2 / \epsilon}}{\left(1+\omega^{\prime}\right)^{1 / \epsilon+\lambda}} f_{2}\left(\omega^{\prime}\right) h\left(\omega^{\prime}\right)$

is regular at $\omega^{\prime}=1$. If $\operatorname{Re} \lambda-\frac{1}{\epsilon}>-1$, we can write

$$
\begin{aligned}
& -c_{3} f_{3}(\omega) \int_{0}^{\omega} \mathrm{d} \omega^{\prime}\left(1-\omega^{\prime}\right)^{\lambda-1 / \epsilon} H\left(\omega^{\prime}\right) \\
& =-c_{3} f_{3}(\omega) \int_{0}^{1} \mathrm{~d} \omega^{\prime}\left(1-\omega^{\prime}\right)^{\lambda-1 / \epsilon} H\left(\omega^{\prime}\right) \\
& +c_{3} \hat{f}_{3}(\omega) \int_{\omega}^{1} \mathrm{~d} \omega^{\prime}\left(\frac{1-\omega^{\prime}}{1-\omega}\right)^{\lambda-1 / \epsilon} H\left(\omega^{\prime}\right) .
\end{aligned}
$$

The second part is regular at $\omega=1$ and the singular first part is canceled by the choice

$a_{1}[h]=\int_{0}^{1} \mathrm{~d} \omega^{\prime}\left(1-\omega^{\prime}\right)^{\lambda-1 / \epsilon} H\left(\omega^{\prime}\right)$.

We note that this result is valid also for $\lambda=\frac{1}{\epsilon}+n, n \in \mathbb{N}$, where $f_{3}(\omega)$ instead of being of the form (36) shows a logarithmic singularity.

If $-n>\operatorname{Re} \lambda-\frac{1}{\epsilon}>-n-1, n \in \mathbb{N}$, we carry through $n$ subtractions of $H\left(\omega^{\prime}\right)$ at $\omega^{\prime}=1$, defining

$\left[H\left(\omega^{\prime}\right)\right]_{n}=H\left(\omega^{\prime}\right)-\sum_{j=0}^{n-1} H_{j}\left(1-\omega^{\prime}\right)^{j}$,

so that $\left[H\left(\omega^{\prime}\right)\right]_{n} \sim$ const $\left(1-\omega^{\prime}\right)^{n}$. A short calculation shows that the singular part of $\int G h$ is canceled by the choice

$$
\begin{aligned}
a_{1}[h]= & \int_{0}^{1} \mathrm{~d} \omega^{\prime}\left(1-\omega^{\prime}\right)^{\lambda-1 / \epsilon}\left[H\left(\omega^{\prime}\right)\right]_{n} \\
& +\sum_{j=0}^{n-1} \frac{H_{j}}{\lambda-\frac{1}{\epsilon}+j+1} .
\end{aligned}
$$

The expressions above clearly remain valid when $\frac{1}{\epsilon}-\operatorname{Re} \lambda=n$, except when $\frac{1}{\epsilon}-\lambda=n$, a positive integer.

When $\frac{1}{\epsilon}-\lambda=n$ is a positive integer, from well-known theory [47] for regular singular points, instead of (31), the 
solutions $f_{1}$ and $f_{2}$ as defined earlier must have the following local representation near $\omega=1$ :

$f_{1}(\omega)=C_{1}(1-\omega)^{n} B_{1}(\omega) \log (1-\omega)+B_{2}(\omega)$,

$f_{2}(\omega)=(1-\omega)^{n} B_{1}(\omega)$,

where $B_{1}$ and $B_{2}$ are analytic at $\omega=1$. If $f_{1}$ and $f_{2}$ are independent, as they are when $\lambda$ is not in the discrete spectrum, then $C_{1}(\epsilon, \lambda)$ $\neq 0$.

We now define

$H(\omega)=B_{2}(\omega)(1+\omega)^{n-2 / \epsilon} \omega^{2 / \epsilon} h(\omega)$,

while $H_{j}$ is still defined by the expression (41). It is also convenient to define

$Q(\omega)=B_{1}(\omega)(1+\omega)^{n-2 / \epsilon} \omega^{2 / \epsilon} h(\omega)$,

Note that each of $H$ and $Q$ are analytic at $\omega=1$. Straight forward calculation based on (34) shows that the possibly singular part of $g(\omega)$ at $\omega=1$ is given by

$$
\begin{aligned}
& -\frac{H_{n-1}}{C} \ln (1-\omega) B_{1}(\omega)(1-\omega)^{n} \\
& \quad+f_{1}(\omega)\left(a_{1}-\int_{0}^{1} \mathrm{~d} \omega^{\prime} \frac{\omega^{\prime 2 / \epsilon} f_{2}\left(\omega^{\prime}\right) h\left(\omega^{\prime}\right)}{C(\epsilon, \lambda)\left(1-\omega^{\prime}\right)^{n}\left(1+\omega^{\prime}\right)^{2 / \epsilon-n}}\right) \\
& \quad+\frac{C_{1} f_{2}(\omega)}{C} \int_{1}^{\omega} \mathrm{d} \omega^{\prime} Q\left(\omega^{\prime}\right) \ln \frac{1-\omega^{\prime}}{1-\omega} .
\end{aligned}
$$

The last term is analytic at $\omega=1$. The singularity vanishes if we choose

$a_{1}=\frac{H_{n-1}}{C_{1} C}+\int_{0}^{1} \mathrm{~d} \omega^{\prime} \frac{\omega^{\prime 2 / \epsilon} f_{2}\left(\omega^{\prime}\right) h\left(\omega^{\prime}\right)}{C(\epsilon, \lambda)\left(1-\omega^{\prime}\right)^{n}\left(1+\omega^{\prime}\right)^{2 / \epsilon-n}}$.

For any $\lambda$ for which $C(\lambda, \epsilon) \neq 0$, using the explicit expression (34) with $a_{1}$ determined from (40), (42) or (47), whatever the case may be, we have in the domain $\mathcal{V}$,

$\|g\|_{\infty} \leq C\|h\|_{\infty}$,

This conclusion follows from observing the properties of the integrand and noting that the $H_{j}, j=1, \ldots n$ are bounded by some multiples of $\sup _{\omega \in \mathcal{V}}|h(\omega)|$, since they involve only a finite number of derivatives of $h$ at $\omega=1$. Since the boundary $\partial \mathcal{V}$ is at a finite distance from $\omega=1$, the derivatives $\left.\partial_{\omega}^{j} h\right|_{\omega=1}$ by Cauchy's theorem are bounded by $b_{j} \sup _{\omega \in \mathcal{V}}|h(\omega)|$ where $b_{j}$ is independent of $h$. Hence, we have shown ${ }^{7}$ that $\lambda$ is in the spectrum only if $C(\lambda, \epsilon)=0$, i.e. we can only have a discrete spectrum in this problem.

\section{Absence of eigenvalues with positive real part and of purely imaginary eigenvalues}

\subsection{Purely positive eigenvalues}

Real eigenvalues $\lambda>0$ easily are excluded. Substituting into Eq. (28) the power series

$\beta_{\lambda}^{(\epsilon)}(\omega)=\sum_{k=0}^{\infty} b_{k} \omega^{k}$,

which converges for $|\omega|<1$ due to the location of the regular singular points, we find the recursion relation

$b_{k}=2 \lambda \frac{1+\epsilon k}{k(2+\epsilon k)} b_{k-1}+\epsilon \frac{k-2}{2+\epsilon k} b_{k-2}$ for $k \geq 2$,

\footnotetext{
7 Note that by definition, $\lambda$ is not in the spectrum if the resolvent $L^{-1}$ is bounded.
}

and

$b_{1}=2 \lambda \frac{1+\epsilon}{2+\epsilon} b_{0}$

We choose $b_{0}=1$ as initial value.

For $\lambda>0$, evidently all $b_{k}$ are positive, and $b_{k}$ obeys the bound $b_{k}>\epsilon \frac{k-2}{2+\epsilon k} b_{k-2}$,

and therefore

$b_{k}>\frac{\Gamma\left(\frac{k}{2}\right)}{\Gamma\left(1+\frac{1}{\epsilon}+\frac{k}{2}\right)}$ const. $>0$.

For $k \gg 1 / \epsilon$, this yields the lower bound

$b_{k}>$ const $k^{-1-1 / \epsilon}$,

which shows that a sufficiently high derivative of $\beta_{\lambda}^{(\epsilon)}(\omega)$ (49) diverges for $\omega=1$, which contradicts the regularity requirement.

\subsection{Eigenvalues with positive real part}

To eliminate eigenvalues $\lambda=\mu+\mathrm{i} v$ with $\mu>0$ needs more refined arguments. We first derive an inequality replacing (52) above. Motivated by (52), we rewrite the recursion relation (50) in terms of

$c_{k}=\frac{\Gamma\left(1+\frac{1}{\epsilon}+\frac{k}{2}\right)}{\Gamma\left(\frac{k}{2}\right)} b_{k}, \quad k \geq 1$.

This yields

$c_{k}=\lambda g_{k} c_{k-1}+c_{k-2}, \quad k \geq 3$,

where

$g_{k} \equiv \frac{2(1+\epsilon k) \Gamma\left(\frac{k-1}{2}\right) \Gamma\left(1+\frac{1}{\epsilon}+\frac{k}{2}\right)}{k(2+\epsilon k) \Gamma\left(\frac{k}{2}\right) \Gamma\left(\frac{1}{2}+\frac{1}{\epsilon}+\frac{k}{2}\right)}$

$$
=\frac{2}{k}\left(1+\mathcal{O}\left(\frac{1}{k}\right)\right) \text {. }
$$

We now multiply (54) by $c_{k-1}^{*}$ and take the real part. With the notation

$r_{k}=\operatorname{Re}\left\{c_{k} c_{k-1}^{*}\right\}$,

we get the relation

$r_{k}=\mu g_{k}\left|c_{k-1}\right|^{2}+r_{k-1}, \quad k \geq 3$.

Since $\mu>0$ and

$$
\begin{aligned}
r_{2} & =\operatorname{Re}\left\{c_{2} c_{1}^{*}\right\} \\
& =2 \mu|\lambda|^{2} \frac{(1+\epsilon)(1+2 \epsilon)}{(2+\epsilon)^{2}} \frac{\Gamma\left(\frac{3}{2}+\frac{1}{\epsilon}\right) \Gamma\left(2+\frac{1}{\epsilon}\right)}{\Gamma\left(\frac{1}{2}\right)} \\
& >0,
\end{aligned}
$$

the $r_{k}$ form an increasing series of positive numbers bounded by $r_{k} \geq r_{2}>0$.

The recursion relation (57) formally is solved as

$r_{k}=\mu \sum_{j=3}^{k} g_{j}\left|c_{j-1}\right|^{2}+r_{2}, \quad k \geq 3$.

Using now the relation

$\left|c_{k}\right|^{2}+\left|c_{k-1}\right|^{2}=2 r_{k}+\left|c_{k}-c_{k-1}\right|^{2} \geq 2 r_{k}$,

we find the bound

$\frac{\left|c_{k}\right|^{2}+\left|c_{k-1}\right|^{2}}{2} \geq \mu \sum_{j=3}^{k} g_{k}\left|c_{j-1}\right|^{2}+r_{2}$. 
We note that this bound is positive and increases monotonically.

We now recall that by definition of the eigenfunctions, the only singularity in the complex plane is of the form

$(1+\omega)^{1 / \epsilon+\lambda} f(\omega)$,

where $f(\omega)$ is regular in $|\omega| \leq 1$, including $\omega=-1$. A standard result on the relation of power series coefficients to the closest complex singularity (see the Appendix) is, that the asymptotic behavior of $b_{k}$ of the expansion (49) satisfies

$\left|b_{k}\right| \sim B_{\infty} k^{-\mu-1 / \epsilon-1}$,

where $B_{\infty}$ is some constant. In view of (53), this implies

$\left|c_{k}\right| \sim$ const $2^{-1-1 / \epsilon} k^{-\mu} \rightarrow 0$

for $k \rightarrow \infty$ which contradicts the bound (60). We thus conclude that there are no eigenvalues $\lambda$ with $\operatorname{Re} \lambda>0$.

\subsection{Purely imaginary eigenvalues}

Now consider the possibility of a purely imaginary eigenvalue $\lambda=\mathrm{i} v$ with $v$ real. From the recursion relation, it is clear that $v=0$ corresponds to the translation mode. So we only consider the case $v \neq 0$. From complex conjugation symmetry, it is clear that if $\lambda=\mathrm{i} v$ is an eigenvalue, so is $\lambda=-\mathrm{i} \nu$. Therefore, we may assume without any loss of generality that $v>0$.

We introduce

$\hat{b}_{k}=i^{-k} b_{k}$.

The recursion relation (50) takes the form

$\hat{b}_{k}=2 v \frac{1+\epsilon k}{k(2+\epsilon k)} \hat{b}_{k-1}-\epsilon \frac{k-2}{2+\epsilon k} \hat{b}_{k-2}$,

$\hat{b}_{0}=1, \quad \hat{b}_{-1}=0$,

which shows that $\hat{b}_{k}$ is real for any $k$. Thus the function

$\hat{\beta}(\hat{\omega})=\sum_{k=1}^{\infty} \hat{b}_{k} \hat{\omega}^{k}=\beta_{\lambda}^{(\epsilon)}(\omega), \quad \hat{\omega}=\mathrm{i} \omega$,

is real for real $\hat{\omega}$, and the reflection principle guarantees

$\hat{\beta}^{*}\left(\hat{\omega}^{*}\right)=\hat{\beta}(\hat{\omega})$.

Thus $\hat{\beta}(\hat{\omega})$ either is singular both at $\hat{\omega}= \pm \mathrm{i}$, corresponding to $\omega=\mp 1$, or is entire. When $\lambda=\mathrm{i} v$ is some eigenvalue, we cannot have a singularity at $\hat{\omega}=-\mathrm{i}$ and so $\hat{\beta}(\hat{\omega})$ must be entire. We will now show that this is impossible.

First, note from the recursion relation that $\hat{\beta}(\hat{\omega})$ cannot be a polynomial since if $b_{k}=0=b_{k-1}$, then so must $b_{k-2}$ and all the previous coefficients. Choose $k_{0}$ so large that for $k \geq k_{0} \geq 4$

$\frac{\epsilon k}{1+\epsilon(k+2)} \geq \frac{1}{2}, \quad \frac{2+\epsilon(k+2)}{\epsilon k} \leq 2$.

We choose a specific $\rho$ large enough that

$\rho \geq 4, \quad \frac{\rho}{2 v}>4$.

Since $\sum_{k=k_{0}}^{\infty} \hat{b}_{k} \hat{\omega}^{k}$ is an entire function, it follows that for any $k_{0}$, there exists a constant $M>0$ so that

$\rho^{k}\left|\hat{b}_{k}\right| \leq M$ for $k \geq k_{0}$.

We redefine $M$ in the relation (66) to be the least upper-bound for $\rho^{k}\left|\hat{b}_{k}\right|$. Note that since $\hat{\beta}(\hat{\omega})$ is not a polynomial, $M$ cannot be zero. We now introduce

$d_{k}=\frac{\rho^{k} \hat{b}_{k}}{M}$ and rewrite recursion relation for $k \geq k_{0}+2$ as

$d_{k}=2 v \frac{1+\epsilon k}{k(2+\epsilon k)} \rho d_{k-1}-\epsilon \frac{k-2}{2+\epsilon k} \rho^{2} d_{k-2}$.

The bound (66) translates into

$\left|d_{k}\right| \leq 1$ for $k \geq k_{0}$.

Solving (68) for $d_{k-1}$, and shifting the indices $k \rightarrow k+2$, we obtain for $k \geq k_{0}$

$d_{k+1}=\frac{\rho(k+2)}{2 v} \frac{\epsilon k}{1+\epsilon(k+2)}\left[d_{k}+\left(\frac{2+\epsilon(k+2)}{\epsilon k \rho^{2}} d_{k+2}\right)\right]$

Since $M$ was the least upper bound for $\rho^{k} \hat{b}_{k}$, it follows that there exists some $k_{*} \geq k_{0}$ so that

$\left|d_{k_{*}}\right| \geq \frac{1}{2}$

On using (64), (65) and $\left|d_{k_{*}+2}\right| \leq 1$, it follows from (70) that

$\left|d_{k_{*}+1}\right| \geq\left(k_{*}+2\right)\left[1-\frac{1}{4}\right]>1$

which is inconsistent with (69).

We note that this argument not only excludes imaginary eigenvalues, but it also shows that except for the trivial translation mode $\beta_{0}^{(\epsilon)}(\omega) \equiv 1$ corresponding to $\lambda=0$, there are no eigenfunctions regular at all three points $\omega=0, \pm 1$. This follows from simply replacing $v$ by $-i \lambda$ (or $|\lambda|$, respectively) in the above analysis without necessarily restricting $\lambda$ to be imaginary.

\section{Calculation of negative eigenvalues for $\epsilon>0$}

We now concentrate on the infinite discrete set of negative eigenvalues $\lambda_{n}(\epsilon)$ which continue the eigenvalues $\lambda_{n}(1)=-n$ found in [35,36]; here the general case of $\epsilon>0$ is considered while the limit $\epsilon \downarrow 0$ is subject of Section 6 .

Observing the parametric dependence of the operator $L$ in (29) on $\epsilon$ and $\lambda$, any solution $\beta$ of the homogeneous equation $L(\epsilon, \lambda) \beta=0$ is analytic in $\lambda$ and $\epsilon$, with the possible exception of $\epsilon=0$. Therefore the Wronskian of any two solutions is an analytic function of $(\lambda, \epsilon)$, except at $\epsilon=0$. Since each eigenvalue $\lambda_{n}(\epsilon)$ is determined as a zero of a particular Wronskian, it follows that it will change continuously with $\epsilon$, except at $\epsilon=0$. Since eigenvalues $\left\{\lambda_{n}(\epsilon)\right\}_{n}$ are all real at $\epsilon=1$, as $\epsilon$ is decreased continuously from 1 towards 0 , the only way eigenvalues can become complex is through collision of erstwhile real eigenvalues, i.e., through the existence of a higher order zero of the Wronskian for some $\epsilon$. Such collisions are not observed in our numerical calculation, consistent with the fact that all eigenspaces are onedimensional, as is obvious from the behavior of $\beta_{\lambda}^{(\epsilon)}(\omega)$ for $\omega \rightarrow 0$ (30). This suggests that eigenvalues with negative real parts and nonvanishing imaginary part are not possible, and therefore they will not be considered in the ensuing.

For the present problem the calculation of $\lambda_{n}(\epsilon)$ as zeros of the Wronskian is feasible only for $\epsilon$ not too small. Relying on the numerical solution of the $\operatorname{ODE}(28)$, with decreasing $\epsilon$ this method rapidly breaks down since for $|\lambda| \ll 1 / \epsilon$, the second independent solution near $\omega=1$ shows only a very weak singularity, cf. Eq. (31). It therefore needs extreme numerical precision to determine reliably the solution that is regular at $\omega=1$.

To circumvent this problem, we note that in the parameter space spanned by $(\epsilon, \lambda)$, there exist special points where both independent solutions of Eq. (31) are regular at $\omega=1$. These points are found on curves

$\lambda=\frac{1}{\epsilon}-m, \quad \frac{1}{\epsilon}<m \in \mathbb{N}$, 
where the general solution near $\omega=1$ can be written as [47]

$$
\begin{aligned}
\beta_{\lambda}^{(\epsilon)}(\omega)= & c_{1}(\omega-1)^{m} B_{1}(\omega) \\
& +c_{2}\left(A(\epsilon)(1-\omega)^{m} B_{1}(\omega) \ln (\omega-1)+B_{2}(\omega)\right) .
\end{aligned}
$$

Here $c_{1,2}$ are arbitrary constants, $B_{1,2}(1)=1$ and both $B_{1}(\omega)$ and $B_{2}(\omega)$ are regular at $\omega=1$. At the zeros $\epsilon_{0}$ of $A(\epsilon)$, the singularity vanishes. For the corresponding $\lambda=1 / \epsilon_{0}-m$, both independent solutions are regular at $\omega=1$. Therefore solutions regular both at $\omega=0$ and at $\omega=1$ can be constructed, and $\lambda$ is an eigenvalue for the particular value $\epsilon_{0}$. In our case, these special points in the $(\epsilon, \lambda)$-plane can be determined as roots of polynomials in $\epsilon$ with integer coefficients; and therefore they can be determined with unlimited numerical precision.

We base the calculation on the formal Taylor expansion about $\omega=1$ :

$\beta_{\lambda}^{(\epsilon)}(\omega)=\sum_{k=0}^{\infty} d_{k}(1-\omega)^{k}$.

The ODE (28) yields the recursion relation

$d_{k}=\frac{\epsilon k(k-2) d_{k-2}-[2 \lambda(1+\epsilon k)+3 \epsilon k(k-1)] d_{k-1}}{2 k(1-\epsilon(\lambda+k))}$

for $k \geq 1$,

where we take $d_{0}=1, d_{-1}=0$. For $\lambda$ as in (73) and for $k=$ $m$, the denominator vanishes and for general $\epsilon$ the ansatz (75) breaks down, showing that $\beta_{\lambda}^{(\epsilon)}(\omega)$ picks up the singular part with $A(\epsilon) \neq 0$ in (74). However, if the numerator in Eq. (76) vanishes, the solution stays regular.

In evaluating this condition, it is preferable to rewrite the recursion relation (76) in terms of

$P_{k}(\epsilon)=\epsilon^{k} k !\left(\prod_{j=1}^{k}(1-\epsilon(\lambda+j))\right) d_{k}$,

with $\lambda=1 / \epsilon-m$ inserted. This yields

$$
\begin{aligned}
P_{k}(\epsilon)= & \epsilon^{4} \frac{k}{2}(k-1)(k-2)(m-k+1) P_{k-2}(\epsilon) \\
& -\left[1+\epsilon(k-m)+\epsilon^{2} \frac{k}{2}(3 k-2 m-3)\right] P_{k-1}(\epsilon)
\end{aligned}
$$

with $P_{-1}(\epsilon)=0$ and $P_{0}(\epsilon)=1 . P_{k}(\epsilon)$ is a polynomial in $\epsilon$ of degree $2 k$, with integer coefficients. Any real zero $\epsilon_{0}$ of $P_{m}(\epsilon)$ yields an eigenvalue $\lambda=1 / \epsilon_{0}-m$.

Using this approach we determined the 11 largest negative eigenvalues for values of $m$ ranging from $m=2$ to $m=2000$, the latter value corresponding to $\epsilon \approx 5 \times 10^{-4}$. We note that the higher coefficients of $P_{m}(\epsilon)$ with increasing $m$ become extremely large, but as function of $\epsilon, P_{m}(\epsilon)$ oscillates around zero with an amplitude that for large $m$ becomes extremely small in the range of interest $0<m-1 / \epsilon=\mathcal{O}(1)$. For $m=2000$ this amplitude is of the order $10^{-1000}$. Nevertheless the zeros of $P_{m}(\epsilon)$ can be determined with arbitrary precision since the coefficients of $P_{m}(\epsilon)$ are integers, exactly determined from the recursion relation (78). Our results are shown in Fig. 1 in a double logarithmic plot. For $n$ fixed, $\left|\lambda_{n}(\epsilon)\right|$ is seen to decrease with decreasing $\epsilon$. For $\epsilon \downarrow 0$, the results suggest the behavior

$\lambda_{n}(\epsilon)=-\alpha_{n} \epsilon^{1 / 2}(1+\mathcal{O}(\epsilon))$,

reflecting the fact that the terms in $\mathcal{L}_{\epsilon}(29)$ that involve second derivatives, for $\epsilon \downarrow 0$ play the role of singular perturbations. The coefficients $\alpha_{n}$ extracted from our data are collected in the middle column of Table 1 . In the next section we consider the limit $\epsilon \downarrow 0$ more closely.

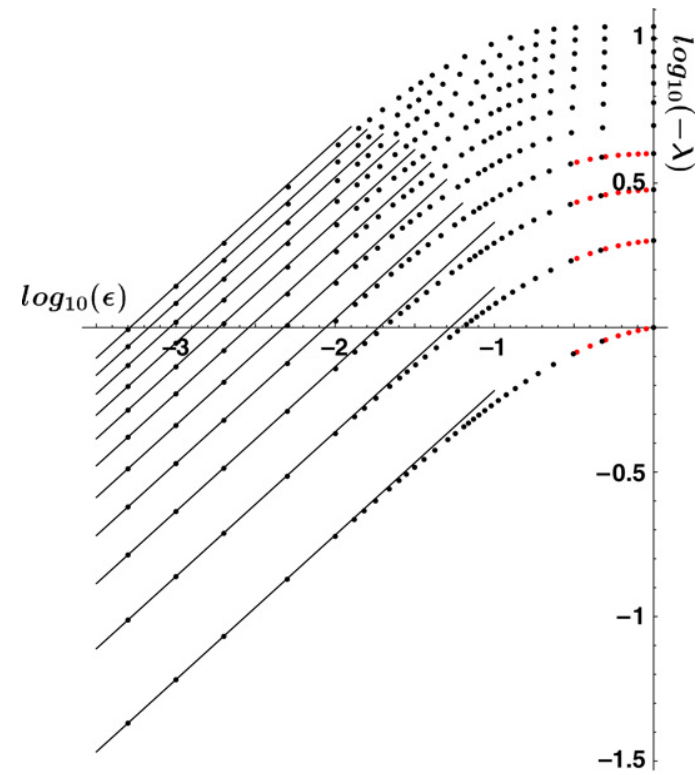

Fig. 1. Double logarithmic plot of the eigenvalues $\lambda_{n}(\epsilon), n=1, \ldots, 11$ (from bottom to top) as a function of $\epsilon$ in the range $5 \times 10^{-4} \leq \epsilon \leq 1$. The black dots indicate discrete eigenvalues of the form (73), gray (online: red) dots give eigenvalues calculated from the Wronskian. The lines are interpolations of the form (79). The coefficients $\alpha_{n}$ determined from these lines can be found in Table 1.

\section{Table 1}

The coefficients $\alpha_{n}$ for $n=1, \ldots, 11$ in the eigenvalue presentation $\lambda_{n}(\epsilon)=$ $-\alpha_{n} \epsilon^{1 / 2}(1+\mathcal{O}(\epsilon))$ from Eq. (79). The middle column is extrapolated from Section 5 and Fig. 1. The right column is from Section 6.1.

\begin{tabular}{rcc}
\hline$n$ & $\alpha_{n}$ from Section 5 & $\alpha_{n}$ from Section 6.1 \\
\hline 1 & 1.9131 & 1.91 \\
2 & 4.3516 & 4.35 \\
3 & 7.3107 & 7.31 \\
4 & 10.7188 & 10.72 \\
5 & 14.5249 & 14.5 \\
6 & 18.6916 & \\
7 & 23.1907 & \\
8 & 27.9992 & \\
9 & 33.0985 & \\
10 & 38.4732 & \\
11 & 44.1103 & \\
\hline
\end{tabular}

\section{The eigenvalues for $\epsilon \downarrow 0$}

\subsection{Eigenvalues with low indices}

According to Eq. (31), the general solution of the ODE (28) at $\omega=1$ develops a singularity that becomes arbitrarily weak if $\epsilon$ tends to zero. As a consequence, representing the special solution $\beta_{\text {reg }}(\omega)$ regular at $\omega=0$ as a power series in $\epsilon$ and imposing regularity at $\omega=1$ does not put any constraint on the eigenvalue $\lambda$. To show this we write $\beta_{\text {reg }}(\omega)$ in the form

$\beta_{\text {reg }}(\omega)=\mathrm{e}^{\lambda \omega} \sum_{j=0}^{\infty} \epsilon^{j} f_{j}(\omega)$,

imposing the normalization condition

$\beta_{\text {reg }}(0)=1$,

i.e.

$f_{0}(0)=1, \quad f_{j}(0)=0, \quad j \geq 1$.

Substituting this ansatz into Eq. (28) we find

$f_{0}(\omega) \equiv 1$ 


$$
\begin{aligned}
f_{j}(\omega)= & -\frac{1}{2}\left(1-\omega^{2}\right) \omega \partial_{\omega} f_{j-1}(\omega)+\lambda \omega^{3} f_{j-1}(\omega) \\
& +\frac{\lambda}{2} \int_{0}^{\omega} \mathrm{d} x\left(1+\lambda x-3 x^{2}+\lambda x^{3}\right) f_{j-1}(x) \quad \text { for } j \geq 1 .
\end{aligned}
$$

Evidently $f_{j}(\omega)$ is a polynomial in $\omega$ of degree $4 j$. Thus $\beta_{\text {reg }}(\omega)$, Eq. (80), evaluated to arbitrary finite order in $\epsilon$, is analytic at $\omega=1$ for arbitrary $\lambda$. This breakdown of an approach based on a simple expansion in the regularizing parameter $\epsilon$ is a well known feature of such moving boundary problems, both in steady state (see for instance [8]) and linear stability analysis (see for instance [12]).

For later use we note the expression for the low order terms:

$$
\begin{aligned}
f_{1}(\omega)= & \frac{\lambda \omega}{2}\left(1+\omega^{2}\right)+\frac{\lambda^{2} \omega^{2}}{4}\left(1+\frac{1}{2} \omega^{2}\right), \\
f_{2}(\omega)= & -\frac{\lambda \omega}{4}\left(1+2 \omega^{2}-3 \omega^{4}\right)+\frac{\lambda^{2} \omega^{2}}{8}\left(-1+3 \omega^{2}+5 \omega^{4}\right) \\
& +\frac{\lambda^{3} \omega^{3}}{16}\left(2+\frac{23}{5} \omega^{3}+\frac{15}{7} \omega^{4}\right) \\
& +\frac{\lambda^{4} \omega^{4}}{32}\left(1+\omega^{2}+\frac{\omega^{4}}{4}\right) .
\end{aligned}
$$

We also note the singular solution of Eq. (28), resulting from a WKB-analysis

$\beta_{\text {sing }}(\omega)=\left(1-\frac{1}{\omega^{2}}\right)^{1 / \epsilon}\left(\frac{1+\omega}{1-\omega}\right)^{\lambda} \mathrm{e}^{-\lambda \omega}(1+\mathcal{O}(\epsilon))$.

This solution picks up the singularities both at $\omega=0$ and at $\omega= \pm 1$.

The above analysis demonstrates that for $\epsilon \downarrow 0$ the eigenvalues $\lambda$ can be determined only by an analysis going beyond all orders in $\epsilon$. Indeed, taking into account the behavior $\lambda \sim \epsilon^{1 / 2}$ suggested by Section 5 , it is evident that the individual terms of the asymptotic expansion (80) become of order 1 for $|\omega| \sim \epsilon^{-1 / 2}$ and consequently the expansion becomes invalid. Since, however, the eigenfunctions $\beta_{\lambda}^{(\epsilon)}(\omega)$ by definition are holomorphic in the right half $\omega$ plane, this suggests to analyze the region $|\omega| \sim \epsilon^{-1 / 2}$ more closely.

We therefore introduce the scaled variable

$\gamma=\frac{1}{\epsilon^{1 / 2} \omega}$

and the notation

$\lambda=-\alpha \epsilon^{1 / 2}$,

$\beta_{\lambda}^{(\epsilon)}(\omega(\gamma))=g(\gamma)$.

With these substitutions, the operator $L(\epsilon, \lambda)$ from (29) takes the form

$L(\epsilon, \lambda)=\frac{\epsilon^{1 / 2}}{2} \gamma\left(L_{0}(\alpha)-\epsilon L_{1}(\alpha)\right)$,

$L_{0}(\alpha)=\partial_{\gamma}^{2}+\left(2 \gamma-\frac{1}{\gamma}\right) \partial_{\gamma}-\frac{2 \alpha}{\gamma}$

$L_{1}(\alpha)=\gamma^{2} \partial_{\gamma}^{2}+(\gamma-2 \alpha) \partial_{\gamma}+\frac{2 \alpha}{\gamma}$.

To leading order in $\epsilon$ we have to discuss the ODE

$L_{0}(\alpha) g(\gamma)=0$.

An asymptotic expansion in powers of $\frac{1}{\gamma}$ yields the solution

$g_{1}(\gamma) \sim 1-\frac{\alpha}{\gamma}+\frac{\alpha^{2}}{2 \gamma^{2}}-\frac{\alpha}{2 \gamma^{3}}\left(1+\frac{\alpha^{2}}{3}\right)+\mathcal{O}\left(\frac{1}{\gamma^{4}}\right)$.

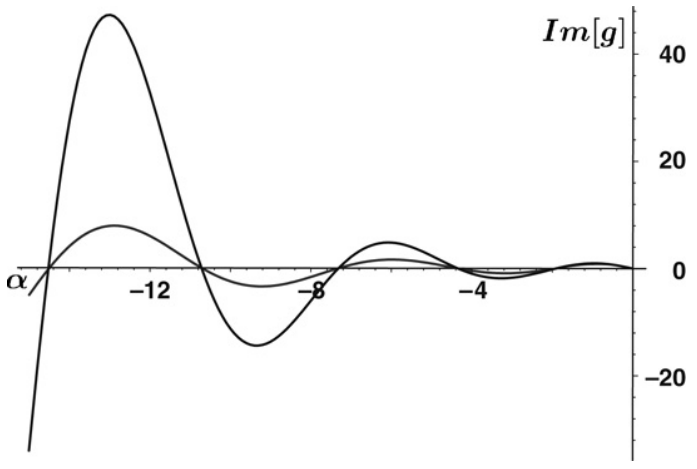

Fig. 2. Im $g(b)$ as a function of $\alpha$ for two different choices of the starting point $\gamma_{0}=b+i L, b=0.1$ and 0.3 ; the zeros of the function determine $\alpha_{n}$.

The second solution, found by balancing the terms $\partial_{\gamma}^{2}$ and $(2 \gamma-$ $\left.\frac{1}{\gamma}\right) \partial_{\gamma}$, takes the form

$g_{2}(\gamma) \sim \mathrm{e}^{-\gamma^{2}}\left(1+\mathcal{O}\left(\frac{1}{\gamma}\right)\right)$.

It is easily seen that in the range

$\epsilon^{-1 / 2} \gg|\gamma| \gg 1$, i.e., $1 \ll|\omega| \ll \epsilon^{-1 / 2}$,

$g_{1}(\gamma)$ matches the regular solution $\beta_{\text {reg }}(\omega)$ given in (80) and (84), whereas $g_{2}(\gamma)$ matches the singular solution $\beta_{\text {sing }}(\omega)$ given in (85).

Evidently $g_{2}(\gamma)$ dominates over $g_{1}(\gamma)$ in the two sectors $\frac{\pi}{4}<$ $|\arg \gamma|<\frac{3}{4} \pi$ for both signs of $\arg \gamma$. We search for eigenfunctions $\beta(\omega)$ matching $g_{1}(\gamma)$ in the complete range $|\arg \gamma|<\frac{\pi}{2}$. Now it is well known that starting deep in the region $\frac{\pi}{4}<\arg \gamma<\frac{\pi}{2}$ with initial conditions taken from the asymptotic expansion (93) and integrating Eq. (92) down into the region $-\frac{\pi}{2}<\arg \gamma<-\frac{\pi}{4}$, we in general will pick up a dominant contribution proportional to $g_{2}(\gamma)$. If, however, $g(\gamma) \in \mathbb{R}$ for $\gamma \in \mathbb{R}$, the Schwarz reflection principle guarantees the absence of such a contribution. Equivalently, we may state that by definition the eigenfunctions $\beta(\omega)$ are real for $\omega \in(0,1)$, and that the absence of the singularity induced cut for $\omega>1$ implies $\beta(\omega) \in \mathbb{R}$ for $\omega>0$. Thus the eigenvalues $\lambda_{n}^{(\epsilon)}=-\alpha_{n} \epsilon^{1 / 2}(1+\mathcal{O}(\epsilon))$ are selected by imposing the constraint $g(\gamma) \in \mathbb{R}$ for $\gamma>0$.

To evaluate this criterion we started at points $\gamma_{0}=b+\mathrm{i} L$, $b=0.1,0.2,0.3, L=20,25,30$ and integrated Eq. (92) down to the real axis along lines $b=$ const. The results for the first 5 positive zeros $\alpha_{n}$ of $\operatorname{Im}[g(b)]$ are given in the right column of Table 1 . They clearly are consistent with the asymptotic results found in Section 5 and presented in the middle column of the same table. We did check that the quoted values to the precision given are insensitive to the starting value $\gamma_{0}$ as long as $L$ is chosen sufficiently large and $b>0$ is not too large or too small. The former causes $\operatorname{Im}[g(b)]$ to be rather small and zeros are harder to detect, while the latter causes numerical inaccuracies due to the singularity of $L_{0}(\alpha)$ at $\gamma=0$. Fig. 2 shows that with different $b$, while $\operatorname{Im}[g(b)]$ itself is different, the zeros match as expected from theory.

\subsection{Eigenvalues with large indices $n$}

Both the approach of the last section and that of Section 5 within reasonable numerical effort yield the asymptotic coefficients $\alpha_{n}$ only for the first few eigenvalues, $\alpha_{n}=\mathcal{O}(1)$. However, in the complementary region $\alpha_{n} \gg 1$, a fully analytical analysis is possible, similar to that performed earlier in the surface tension selection problem for steady Hele-Shaw fingers $[8,11,13]$. We first introduce a Liouville transformation to eliminate the first order derivative in the operator $L_{0}(\alpha)$. With

$g(\gamma)=\gamma^{1 / 2} \mathrm{e}^{-\gamma^{2} / 2} h(\gamma)$, 
Eq. (92) takes the form

$\partial_{\gamma}^{2} h-\left(\gamma^{2}+\frac{2 \alpha}{\gamma}+\frac{3}{4 \gamma^{2}}\right) h=0$.

We now rescale $\gamma$ according to

$\gamma=\alpha^{1 / 3} \chi, \quad h(\gamma(\chi))=q(\chi)$

to find

$\partial_{\chi}^{2} q-\alpha^{4 / 3}\left(\chi^{2}+\frac{2}{\chi}+\frac{3}{4 \alpha^{4 / 3} \chi^{2}}\right) q=0$.

For $\alpha \rightarrow \infty$, a WKB-analysis yields the asymptotic relation

$q(\chi) \sim C_{1} q_{1}(\chi)+C_{2} q_{2}(\chi)$,

$q_{1,2}(\chi)=Q^{-1 / 4}(\chi) \exp \left[ \pm \alpha^{2 / 3} \int_{0}^{\chi} \mathrm{d} \chi^{\prime} Q^{1 / 2}\left(\chi^{\prime}\right)\right]$,

where

$Q(\chi)=\chi^{2}+\frac{2}{\chi}$.

$q_{2}(\chi)$ dominates in the sectors $\frac{\pi}{4}<|\arg \chi|<\frac{\pi}{2}$, but is subdominant on the real axis. Recalling Eqs. (95) and (97), it is easily seen that for large $|\chi|, q_{1}(\chi)$ yields $g_{1}(\gamma)$, Eq. (93), whereas $q_{2}(\chi)$ yields $g_{2}(\gamma)$, Eq. (94). Since the eigenfunctions in the limit $\epsilon \downarrow 0$ in all the right half plane $\operatorname{Re}[\gamma]>0$ must reduce to $g_{1}(\gamma)$, this implies that $C_{1}$ is real whereas $C_{2}$ has to vanish in both sectors $\frac{\pi}{4}<|\arg \chi|<\frac{\pi}{2}$.

Now the WKB-analysis breaks down at the turning points $\chi_{s}$ defined by $Q\left(\chi_{s}\right)=0$, which in the relevant region $\operatorname{Re}[\chi]>0$ yields the two solutions

$\chi_{s}=2^{1 / 3} \mathrm{e}^{ \pm \mathrm{i} \pi / 3}$.

As is well known in asymptotics [48] the coefficients in the asymptotic relation (99) may jump when $\chi$ crosses a Stokes line emerging from a turning point. Thus a vanishing $C_{2}$ in the sector $\frac{\pi}{4}<\arg \chi<\frac{\pi}{2}$ does not imply that $C_{2}$ vanishes on the positive real axis as well. Since, however, the eigenfunctions are real on the real axis, we get the relation

$\operatorname{Im}\left[C_{2}\right]=0$ for $\chi \in \mathbb{R}_{+}$

as a necessary condition fixing the eigenvalues. To evaluate this condition we determine the jump of $C_{2}$ by analyzing the neighborhood of the turning point $\chi_{s}=2^{1 / 3} \mathrm{e}^{+i \pi / 3}$.

Expanding $Q(\chi)$ about $\chi_{s}$,

$Q(\chi)=Q^{\prime}\left(\chi_{s}\right)\left(\chi-\chi_{s}\right)+\mathcal{O}\left(\chi-\chi_{s}\right)^{2}$,

$Q^{\prime}\left(\chi_{s}\right)=\frac{6}{2^{2 / 3}} \mathrm{e}^{\mathrm{i} \pi / 3}$

and rescaling $\chi-\chi_{s}$ according to

$\xi=\alpha^{4 / 9}\left(\frac{6}{2^{2 / 3}}\right)^{1 / 3} \mathrm{e}^{-\mathrm{i} 5 \pi / 9}\left(\chi-\chi_{s}\right)$,

$q(\chi(\xi))=m(\xi)$,

we find that Eq. (98) to leading order in $\alpha$ reduces to the Airy equation

$$
\left(\partial_{\xi}^{2}-\xi\right) m(\xi)=0 .
$$

The phase in Eq. (106) has been chosen such that for $\xi>0$ we enter the region where $C_{2}=0$ in the asymptotic relation (99). This analysis is valid for $\left|\chi-\chi_{s}\right| \gg \alpha^{-4 / 9}$, and using Eq. (104), we find for small $\left|\chi-\chi_{s}\right|$ along the line $\xi>0$

$q(\chi) \sim a \xi^{-1 / 4} \exp \left[-\frac{2}{3} \xi^{3 / 2}\right]$ where the constant $a$ is given by

$$
\begin{aligned}
a= & \alpha^{1 / 9} C_{1}\left(\frac{2^{2 / 3}}{6}\right)^{1 / 6} \mathrm{e}^{-\mathrm{i} 2 \pi / 9} \\
& \times \exp \left[\alpha^{2 / 3} \int_{0}^{\chi_{s}} \mathrm{~d} \chi^{\prime} \sqrt{Q\left(\chi^{\prime}\right)}\right] .
\end{aligned}
$$

This result is valid for $\alpha^{4 / 9} \gg \xi \gg 1$, where it must match the solution of the Airy equation (108). This yields

$m(\xi)=2 \sqrt{\pi} a \operatorname{Ai}(\xi)$.

Continuing this result clockwise around the turning point to negative $\xi$, we find

$$
\begin{aligned}
m(\xi) \sim & a|\xi|^{-1 / 4} \mathrm{e}^{\mathrm{i} \pi / 4}\left(\exp \left[-\frac{2}{3} \mathrm{i}|\xi|^{3 / 2}\right]\right. \\
& \left.-\mathrm{i} \exp \left[\frac{2}{3} \mathrm{i}|\xi|^{3 / 2}\right]\right) \text { for }-\xi \gg 1 .
\end{aligned}
$$

For $\alpha^{4 / 9} \gg-\xi \gg 1$, this again has to match with Eq. (99), which in this region reduces to

$$
\begin{aligned}
q(\chi) \sim & a|\xi|^{-1 / 4} \mathrm{e}^{\mathrm{i} \pi / 4}\left(\exp \left[-\frac{2}{3} i|\xi|^{3 / 2}\right]\right. \\
& +\frac{C_{2}}{C_{1}} \exp \left[-2 \alpha^{2 / 3} \int_{0}^{\chi_{s}} \mathrm{~d} \chi^{\prime} \sqrt{Q\left(\chi^{\prime}\right)}\right] \\
& \left.\times \exp \left[\frac{2}{3} i|\xi|^{3 / 2}\right]\right) .
\end{aligned}
$$

We thus find

$C_{2}=-i C_{1} \exp \left[2 \alpha^{2 / 3} \int_{0}^{\chi_{s}} \mathrm{~d} \chi^{\prime} \sqrt{Q\left(\chi^{\prime}\right)}\right]$.

Since both $C_{1}$ and $C_{2}$ have to be real, this leads to the eigenvalue condition

$\operatorname{Im}\left[2 \alpha^{2 / 3} \int_{0}^{\chi_{s}} \mathrm{~d} \chi^{\prime} \sqrt{Q\left(\chi^{\prime}\right)}\right]=\left(n+\frac{1}{2}\right) \pi, \quad n \in \mathbb{Z}$.

The integral is easily evaluated to yield

$$
\begin{aligned}
\int_{0}^{\chi_{s}} \mathrm{~d} \chi^{\prime} \sqrt{Q\left(\chi^{\prime}\right)} & =2^{2 / 3} \mathrm{e}^{\mathrm{i} \pi / 6} \int_{0}^{1} \mathrm{~d} s \sqrt{\frac{1}{s}-s^{2}} \\
& =2^{2 / 3} \frac{\sqrt{\pi}}{8} \frac{\Gamma\left(\frac{1}{6}\right)}{\Gamma\left(\frac{2}{3}\right)}(\sqrt{3}+i) .
\end{aligned}
$$

Eq. (115) yields the final result

$$
\begin{aligned}
\alpha_{n} & =4\left(\frac{\Gamma\left(\frac{2}{3}\right)}{\Gamma\left(\frac{1}{6}\right)} \sqrt{\pi}\left(n+\frac{1}{2}\right)\right)^{3 / 2} \\
& =1.13254 \ldots\left(n+\frac{1}{2}\right)^{3 / 2} \text { for } n \in \mathbb{N}_{0} .
\end{aligned}
$$

By construction this result is valid in the limit $\alpha_{n} \rightarrow \infty$, i.e., for $n \rightarrow \infty$. However, it turns out to be a good approximation for small $n$ as well. This is illustrated in Fig. 3 where we plot

$\bar{\alpha}_{n}=\frac{\alpha_{n}}{4}\left(\frac{\Gamma\left(\frac{2}{3}\right)}{\Gamma\left(\frac{1}{6}\right)} \sqrt{\pi}\left(n+\frac{1}{2}\right)\right)^{-3 / 2}$,

with $\alpha_{n}, n=1, \ldots, 11$ taken from the second column of Table 1 . Even for $n=2$ the asymptotic result (117) differs from the true eigenvalue by less than $3 \%$. The interpolating curve included in Fig. 3 is given by

$\bar{\alpha}_{n}=1-0.18\left(n+\frac{1}{2}\right)^{-2}$,

indicating that the asymptotic limit (117) is rapidly approached. 


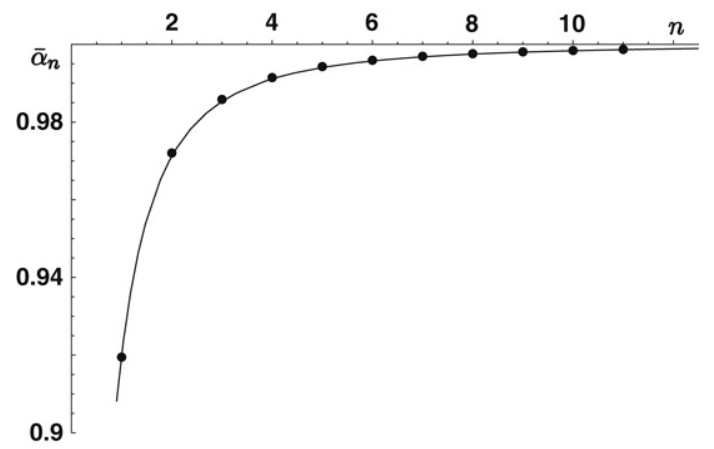

Fig. 3. $\bar{\alpha}_{n}$ from Eq. (119) as a function of $n$, together with the interpolating formula (120). The plot demonstrates the accuracy of the asymptote (117) even for small $n$.

\section{Behavior of low order eigenfunctions for $|\omega| \leq 1$}

As is evident from Eq. (31), for $\omega \rightarrow-1$ the eigenfunctions are singular,

$\beta_{\lambda}^{(\epsilon)}(\omega) \sim(1+\omega)^{\frac{1}{\epsilon}+\lambda}$.

For small $\epsilon$ and $|\lambda| \ll 1 / \epsilon$, this singularity will show up only in derivatives of high order, and the Taylor expansion (49) will converge in the whole physical domain $|\omega| \leq 1$. We therefore can use this expansion together with the recursion relation (50) and (51) to evaluate $\beta_{\lambda}^{(\epsilon)}(\omega)$ for the first few eigenvalues. Outside some neighborhood of $\omega=-1$ these functions are expected to govern the large time behavior of analytic perturbations.

From the discussion of $\beta_{\text {reg }}(\omega)$ in Section 6.1 we expect that in the physical domain $|\omega| \leq 1$ the eigenfunctions for small $\epsilon$ are well approximated by simple exponentials. This indeed is true as illustrated in Fig. 4. Panel 4(a) shows the absolute value $\left|\beta_{\lambda_{1}}^{(\epsilon)}(\omega) \mathrm{e}^{-\lambda_{1} \omega}\right|$ evaluated on the unit circle $\omega=\mathrm{e}^{\mathrm{i} \alpha}$ for a decreasing set of values of $\epsilon$. Evidently this ratio tends to 1 and is not very different from 1 even for $\epsilon=0.1$. Panel 4(b) shows the corresponding phase difference which is found to be very small and to tend to zero with decreasing $\epsilon$. All curves in Fig. 4 are well represented by the expansion (80) evaluated up to order $\epsilon^{2}$. As expected, for fixed $\epsilon$ the approximation

$\beta_{\lambda}^{(\epsilon)}\left(\mathrm{e}^{\mathrm{i} \alpha}\right) \sim \exp (\lambda \cos \alpha+\mathrm{i} \lambda \sin \alpha)$

becomes worse with increasing $\lambda$. This is illustrated in Fig. 5 for $\epsilon=0.1, \lambda_{1}=-0.5462, \lambda_{6}=-4.6086$. Panel 5(a) compares $\operatorname{Re}\left(\ln \beta_{\lambda}^{(\epsilon)}\left(\mathrm{e}^{i \alpha}\right)\right) / \lambda$ with $\cos \alpha$. In the neighborhood of $\alpha=\pi$ the deviation from $\cos \alpha$ increases with increasing $\lambda$, which is a consequence of the singularity of $\omega=-1$. But outside that range the approximation (121) is quite accurate even for $\beta_{\lambda_{6}}^{(1)}$. Panel 5(b) shows the corresponding results for $\operatorname{Im}\left(\ln \beta_{\lambda}^{(\epsilon)}\left(\mathrm{e}^{\mathrm{i} \alpha}\right)\right) / \lambda$.

As pointed out in Section 2.4, we expect that the initial condition $\beta(\omega, 0)$ in some neighborhood of $\omega=1$ can be expanded in terms of eigenfunctions as

$\beta(\omega, 0)=\sum_{k=0}^{\infty} g_{n} \beta_{\lambda_{n}}^{(\epsilon)}(\omega)$,

and that the domain of convergence of this expansion increases with time to ultimately cover $U_{\omega} \backslash\{-1\}$. For small $\epsilon$ and not too large $|\lambda|, \beta_{\lambda}^{(\epsilon)}(\omega)$ is well approximated by $\mathrm{e}^{\lambda \omega}$, and all eigenvalues vanish for $\epsilon \rightarrow 0$. Thus for fixed $\omega$ and $\epsilon \rightarrow 0$, all eigenfunctions tend to 1 . This suggests that for small $\epsilon$ many terms must contribute non-negligibly to the expansion of a generic initial condition. Furthermore the phase of the coefficients $g_{n}$ must vary strongly. With decreasing $\epsilon$ it will need more and larger coefficients to describe a non-trivial general initial condition. These large coefficients imply large transient growth, because in the representation

$\beta(\omega, t)=\sum_{k=0}^{\infty} g_{n} \beta_{\lambda_{n}}^{(\epsilon)}(\omega) \mathrm{e}^{\lambda_{n} t}$,

the factor $\mathrm{e}^{\lambda_{n} t}$ is substantially different for each $n$ when $t=$ $O\left(\epsilon^{-1 / 2}\right)$. The large transient growth restricts the validity of linear stability analysis to a small ball of initial conditions. This is confirmed in part II in the study of the initial value problem.

\section{Conclusion and outlook}

In the introduction we raised the question: Can a kinetic undercooling boundary condition regularize the evolution of a curved interface in a Laplacian growth model? This problem is motivated by the physics of streamer discharges which determine the early evolution of sparks and lightning. We here gave a first answer by analyzing the spectrum of linear perturbations of uniformly translating circles. We proved that for all $\epsilon>$ 0 the spectrum is discrete and that all eigenvalues $\lambda_{n}$, except for $\lambda_{0}=0$, have a negative real part. Thus any infinitesimal perturbation tends to a constant exponentially in time for large time, and asymptotically the circular shape is recovered. For arbitrary regularization parameter $\epsilon>0$ we found an infinite set of negative real eigenvalues $\lambda_{n}(\epsilon)$; they smoothly continue the exact result $\lambda_{n}(1)=-n, n \in \mathbb{N}$, found previously for $\epsilon=1$ [36].

In formulating the eigenvalue problem we had to allow for a singularity of the eigenfunctions at the point $\omega=-1$ at the back of the circle. Therefore an expansion of a regular initial perturbation in terms of eigenfunctions must break down in a neighborhood of $\omega=-1$. For $\epsilon=1$, it was found [36] that the size of this neighborhood with increasing time $\tau$ decreases as $\mathrm{e}^{-\tau}$, and that all the structure of the initial perturbation is convected into this region. In part II [43] of this series of papers we will argue that such behavior is found for all $\epsilon>0$.

Our results suggest that in the framework of linear perturbation theory the evolution of a curved front can be regularized by a kinetic undercooling boundary condition. Linear perturbation theory must break down in the limit $\epsilon \downarrow 0$. In our results this shows up in the asymptotic behavior of the eigenvalues $\lambda_{n}(\epsilon)=-\alpha_{n} \epsilon^{1 / 2}$, where $\alpha_{n} \sim$ const $n^{3 / 2}$ for large $n$, and in the associated behavior $\beta_{\lambda_{n}}^{(\epsilon)}(\omega) \rightarrow \mathrm{e}^{\lambda_{n} \omega} \rightarrow 1$ of the eigenfunctions. This indicates that for small $\epsilon$, the eigenfunction expansion of even a very smooth initial perturbation of small but finite amplitude will contain many terms with large coefficients. Initially these terms almost compensate, but the balance is destroyed by the temporal evolution. Generically this will lead to a strong transient growth of the perturbation which may drive the evolution into the regime where nonlinear effects have to be included. Examples supporting this scenario will be given in part II [43]. For $\epsilon \ll 1$ this mechanism can lead to an instability of the circular shape also against quite small and smooth perturbations.

\section{Acknowledgments}

S. Tanveer acknowledges hospitality at CWI Amsterdam. Additional support was provided by the U.S. National Science Foundation (DMS-0405837, DMS-0733778, DMS-0807266). F. Brau acknowledges a grant of The Netherlands' Organization for Scientific Research NWO within the FOM/EW-program "Dynamics of Patterns". 

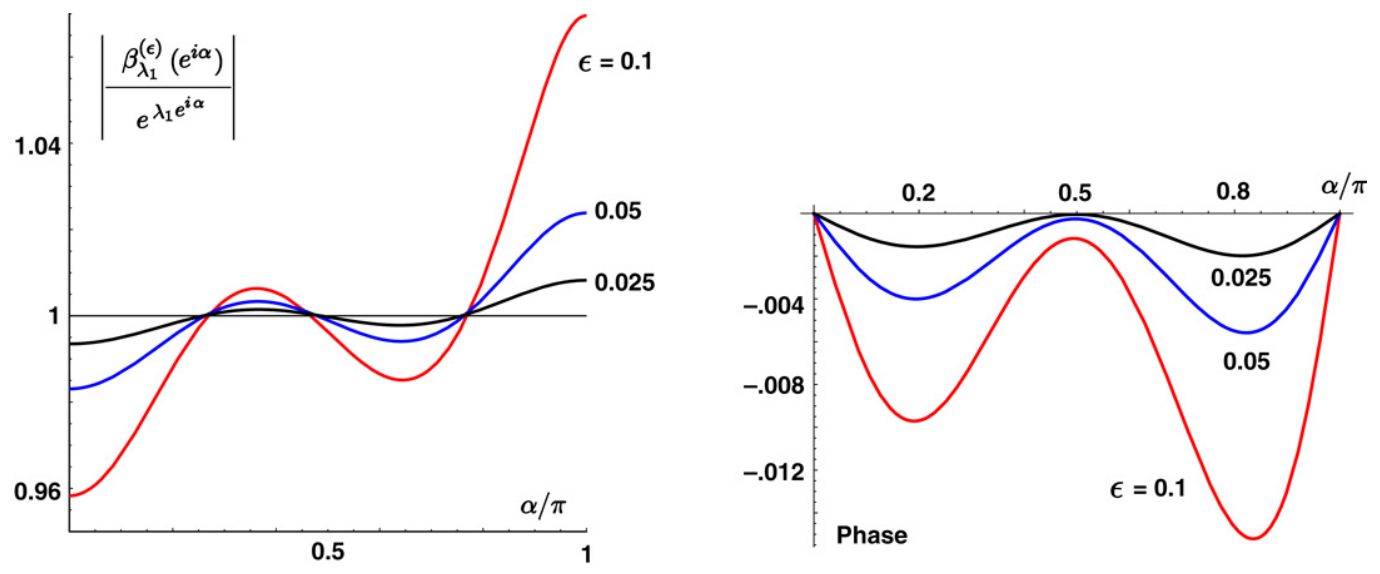

Fig. 4. The ratio $\mathcal{F}=\beta_{\lambda_{1}}^{(\epsilon)}(\omega) / \mathrm{e}^{\lambda_{1} \omega}$ evaluated on the unit circle $\omega=\mathrm{e}^{\mathrm{i} \alpha}$ for $\epsilon=0.1,0.05,0.025$ as indicated. (a) Absolute value $|\mathcal{F}| \operatorname{and}(\mathrm{b}) \operatorname{argument} \operatorname{Arg}(\mathcal{F}) / \pi$ as a function of $\alpha / \pi$.
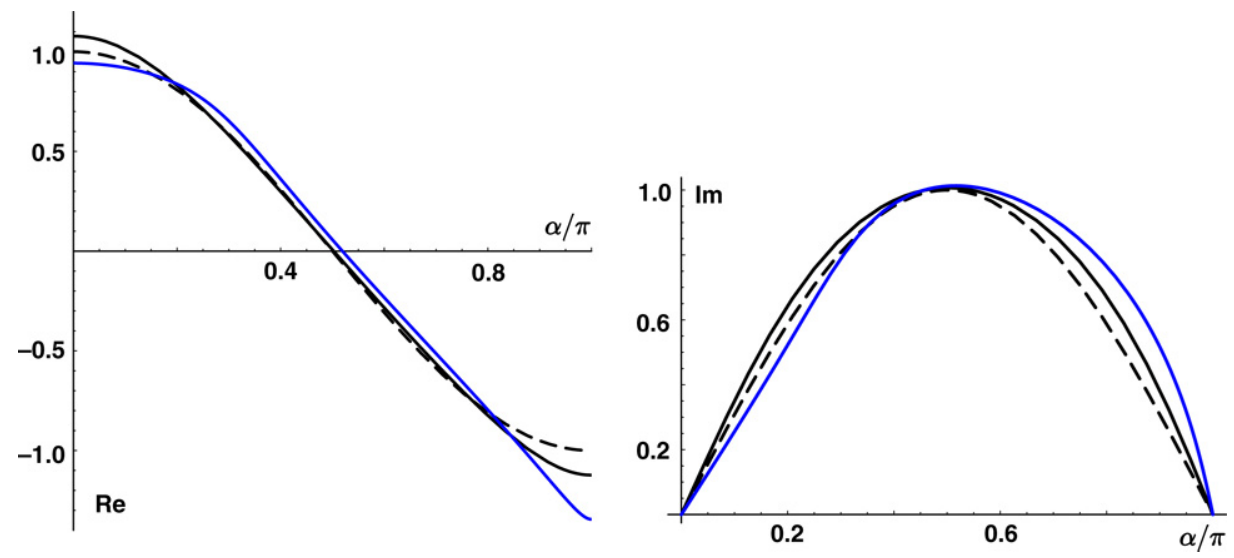

Fig. 5. Real part (a) and imaginary part (b) of the function $g=\ln \left[\beta_{\lambda}^{(\epsilon)}\left(\mathrm{e}^{\mathrm{i} \alpha}\right)\right] / \lambda$ for $\epsilon=0.1$ for $\lambda_{1}$ (black solid line) and $\lambda_{6}$ (blue or gray solid line) as a function of $\alpha / \pi$. The dashed lines show $\cos \alpha$ in (a) or $\sin \alpha$ in (b) for comparison.

\section{Appendix. Asymptotics of Taylor series coefficients and rela- tion to the singularity at $\omega=\omega_{s}$}

Here we prove the following standard results for the sake of completeness.

Theorem. Assume $f(\omega)$ is analytic in $|\omega|<R$, except for a singularity at $\omega=\omega_{s} \neq 0$ (with $\left.\left|\omega_{s}\right|<R\right)$, where

$f(\omega)=f_{s}(\omega)\left(\omega-\omega_{s}\right)^{\alpha}+f_{a}(\omega)$ as $\omega \rightarrow \omega_{s}$.

Here $\alpha$ is not a positive integer, and $f_{s}$ and $f_{a}$ are locally analytic at $\omega=\omega_{s}$ with $f_{s}\left(\omega_{s}\right) \equiv C \neq 0$. Then the Taylor series coefficient $b_{k}$ in the representation

$f(\omega)=\sum_{k=0}^{\infty} b_{k} \omega^{k}$

has the leading order asymptotic behavior

$b_{k} \sim-\frac{C \mathrm{e}^{\mathrm{i} \pi \alpha}}{\pi} \sin (\pi \alpha) \omega_{s}^{-k+\alpha} \frac{\Gamma(\alpha+1)}{k^{\alpha+1}}$.

Proof. We will first assume $\alpha>-1$. We recall the contour integral representation of $b_{k}$

$b_{k}=\frac{1}{2 \pi \mathrm{i}} \oint_{|\omega|=\delta} \frac{f(\omega)}{\omega^{k+1}} \mathrm{~d} \omega$, where $\delta$ is chosen sufficiently small so that $|\omega|=\delta$ contains no singularity of $f(\omega)$ and $\left|\omega_{s}\right|+\delta<R$. We deform the contour into $\int_{C_{0}}+\int_{L_{1}}+\int_{L_{2}}$ as shown in Fig. 6. The contribution from $\int_{C_{0}}$ is easily bounded by $M\left[\left|\omega_{s}\right|+\delta\right]^{-k}$, where $M=\sup _{|\omega|=\left|\omega_{s}\right|+\delta}|f(\omega)|$. Now consider the contribution from $\int_{L_{1}}+\int_{L_{2}}$. It is convenient to introduce a change of variable

$\zeta=\log \frac{\omega}{\omega_{s}}$.

Then it is readily checked that we have

$\int_{L_{1}}+\int_{L_{2}}=\frac{\omega_{s}^{-k}}{2 \pi \mathrm{i}}\left\{\int_{0}^{\delta_{1}}-\int_{0 \mathrm{e} 2 \mathrm{i} \pi}^{\delta_{1} \mathrm{e}^{2 i \pi}}\right\} f(\omega(\zeta)) \mathrm{e}^{-k \zeta} \mathrm{d} \zeta$

where $\delta_{1}=\log \left[1+\frac{\delta}{\left|\omega_{s}\right|}\right]$. Noting that the contribution from $f_{a}$ cancels out between $L_{1}$ and $L_{2}$, we obtain

$\int_{L_{1}}+\int_{L_{2}}=\frac{\omega_{s}^{-k+\alpha}}{2 \pi \mathrm{i}}\left[1-\mathrm{e}^{2 \mathrm{i} \pi \alpha}\right] \int_{0}^{\delta_{1}}\left[\mathrm{e}^{\zeta}-1\right]^{\alpha} f_{s}(\omega(\zeta)) \mathrm{e}^{-k \zeta} \mathrm{d} \zeta$

We note that in the neighborhood of $\zeta=0$,

$\left[\mathrm{e}^{\zeta}-1\right]^{\alpha} f_{s}(\omega(\zeta)) \sim C \zeta^{\alpha}$ 


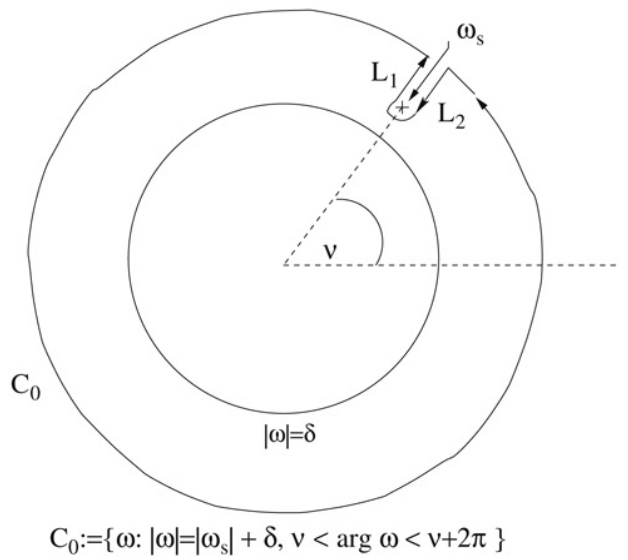

Fig. 6. Contour $\oint_{|\omega|=\delta}$ deformed to $\int_{C_{0}}+\int_{L_{1}}+\int_{L_{2}}$ for evaluation of $b_{k}$.

So, using Watson's Lemma, we obtain

$\int_{L_{1}}+\int_{L_{2}} \sim \frac{C}{2 \pi \mathrm{i}} \omega_{s}^{-k+\alpha}\left[1-\mathrm{e}^{2 \pi i \alpha}\right] k^{-1-\alpha} \Gamma(1+\alpha)$,

from which the Lemma follows since the contribution from $\int_{C_{0}}$ is evidently exponentially small relatively for large $k$.

If $\alpha<-1$, we consider $n$-th iterated integral $I_{n} f$, where $\left[I_{1} f\right](\omega)=\int_{0}^{\omega} f\left(\omega^{\prime}\right) d \omega^{\prime}, I_{2} f=I_{1}\left(I_{1} f\right)$ and so on. Then, it is clear that the singularity of $I_{n} f$ at $\omega=\omega_{s}$ will be of the type $\left(\omega-\omega_{s}\right)^{\alpha+n}$, and we can arrange $\alpha+n>-1$. The argument above can then be repeated for power series coefficients of $I_{n} f$. The Theorem follows on differentiating $n$-times the power series of $I_{n} f$.

Remark. The previous theorem remains valid even when $\alpha$ is a negative integer, provided the product of $\sin (\pi \alpha) \Gamma(1+\alpha)$ is replaced by its finite nonzero limit $\lim _{\alpha \rightarrow-n}$. The validity of the result is easily checked from Taylor expansion of $\frac{1}{\left(\omega-\omega_{s}\right)}$ and its derivatives in terms of a geometric series and its derivatives. Further, since the asymptotic result relies on Watson's Lemma, the condition on analyticity of $f_{s}$ at $\omega=\omega_{s}$ can be weakened to $f_{s}(\omega)=C+\mathcal{O}\left(\left(\omega-\omega_{s}\right)^{\beta}\right)$ for $\beta>0$.

Theorem. If the $k$-th Taylor series coefficient $b_{k}$ of an analytic function $f$ at $\omega=0$ satisfies the following

$b_{k} \sim-\frac{C}{\pi} \mathrm{e}^{\mathrm{i} \pi \alpha} \sin (\pi \alpha) \omega_{s}^{-k+\alpha} \frac{\Gamma(\alpha+1)}{k^{\alpha+1}}(1+O(1 / k))$

for non-integer $\alpha$, then

$f(\omega) \sim C\left(\omega-\omega_{s}\right)^{\alpha}+f_{a}(\omega)$,

where $f_{a}(\omega)$ is regular at $\omega=\omega_{s}$. The same conclusion is valid for $\alpha=-n$, a negative integer, provided $\Gamma(1+\alpha) \sin (\pi \alpha)$ is replaced by its limit as $\alpha \rightarrow-n$, On the other hand, if

$b_{k} \sim-C \omega_{s}^{-k} k^{-1}(1+O(1 / k))$,

then

$f(\omega) \sim C \log \left(1-\frac{\omega}{\omega_{s}}\right)$.

Proof. We will assume for now that $\alpha \in(-1,0)$. Then using the previous Theorem to determine the Taylor series coefficient of $C\left(\omega-\omega_{s}\right)^{\alpha}$, it follows on subtraction that

$g(\omega) \equiv f(\omega)-C\left(\omega-\omega_{s}\right)^{\alpha}=\sum_{k=0}^{\infty} b_{k} \omega^{k}$ will have a Taylor series coefficient $b_{k} \sim$ Const. $\omega_{s}^{-k} k^{-\alpha-2}$ for large $k$ implying that the series is absolutely convergent on $|\omega|=\left|\omega_{s}\right|$. In particular, $g$ is continuous at $\omega=\omega_{s}$, which proves the theorem for $\alpha \in(-1,0)$. If $\alpha$ takes on other ranges of values, we obtain this result by either $n$-times iterative integration from the origin of the power series for $f$, or by differentiating it $n$ times so as to ensure that for non-integer $\alpha, \alpha+n \in(-1,0)$ or $\alpha-n \in$ $(-1,0)$. The result quoted in the Theorem follows by noting that the asymptotics is differentiable as it is valid for $\omega-\omega_{s}$ in a complex sector. The second result follows from noting the explicit Taylor expansion of $\log \left(1-\frac{\omega}{\omega_{s}}\right)$ and from noting that the difference has a series that is absolutely summable and hence the remainder is continuous at $\omega=\omega_{s}$. By using explicit derivatives or integrals of Geometric series, the conclusion (126) holds for negative integer $\alpha=-n$ as well.

\section{References}

[1] D. Kessler, J. Koplik, H. Levine, Patterned selection in fingered growth phenomena, Adv. Phys. 37 (1988) 255.

[2] P. Pelcé, Dynamics of Curved Fronts, Academic, Boston, 1988.

[3] S. Tanveer, The effect of surface tension on the shape of a Hele-Shaw cell bubble, Phys. Fluids 29 (1986) 3537.

[4] D. Bensimon, Stability of viscous fingering, Phys. Rev. A 33 (1986) 1302.

[5] D. Bensimon, L.P. Kadanoff, S. Liang, B.I. Shraiman, C. Tang, Viscous flows in two dimensions, Rev. Modern Phys. 58 (1986) 977.

[6] A.J. DeGregoria, L.W. Schwartz, A boundary integral method for two-phase displacement in Hele-Shaw cells, J. Fluid Mech. 164 (1986) 164.

[7] D. Kessler, H. Levine, Stability of finger patterns in Hele-Shaw cells, Phys. Rev. A 33 (1986) 2632.

[8] R. Combescot, T. Dombre, V. Hakim, Y. Pomeau, A. Pumir, Shape selection for Saffman Taylor fingers, Phys. Rev. Lett. 56 (1986) 2036

[9] S. Howison, Fingering in Hele-Shaw cells, J. Fluid Mech. 167 (1986) 439.

[10] S. Tanveer, P.G. Saffman, Stability of bubbles in a Hele-Shaw cell, Phys. Fluids 30 (1987) 2624.

[11] S. Tanveer, Analytic theory for the selection of symmetric Saffman-Taylor finger in a Hele-Shaw cell, Phys. Fluids 30 (1987) 1589.

[12] S. Tanveer, Analytic theory for the linear stability of Saffman-Taylor finger Phys. Fluids 30 (1987) 2318.

[13] A.T. Dorsey, O. Martin, Saffman Taylor fingers with anisotropic surface tension, Phys. Rev. A 35 (1987) 3989.

[14] S. Tanveer, P.G. Saffman, The effect of finite viscosity ratio on the stability of fingers and bubbles in a Hele-Shaw cell, Phys. Fluids 31 (1988) 3188.

[15] S. Tanveer, Surprises in viscous fingering, J. Fluid Mech. 409 (2000) 273.

[16] S.D. Howison, Complex variable methods in Hele-Shaw moving boundary problems, European J. Appl. Math. 3 (1992) 209.

[17] T. Dombre, V. Hakim, Saffman-Taylor fingers and directional solidification at low velocity, Phys. Rev. A 36 (1987) 2811.

[18] E. Brener, V.I. Melnikov, Pattern selection in two-dimensional dendritic growth, Adv. Phys. 40 (1991) 53.

[19] J.J. Xu, Interfacial wave theory of solidification: Dendritic pattern formation and selection of growth, Phys. Rev. A 43 (1991) 930.

[20] M.D. Kunka, M.R. Foster, S. Tanveer, Dendritic crystal growth for weak undercooling, Phys. Rev. E 56 (1997) 3068.

[21] P.S. Ho, Motion of inclusion induced by a direct current and a temperature gradient, J. Appl. Phys. 41 (1970) 64.

[22] M. Mahadevan, R.M. Bradley, Stability of a circular void in a passivated currentcarrying metal film, J. Appl. Phys. 79 (1996) 6840.

[23] M. Ben Amar, Void electromigration as a moving free-boundary value problem, Physica D 134 (1999) 275.

[24] L.J. Cummings, G. Richardson, M. Ben-Amar, Models of void electro-migration, European J. Appl. Math. 12 (2001) 97.

[25] P. Kuhn, J. Krug, F. Hausser, A. Voigt, Complex shape evolution of electromigration-driven Single-Layer Islands, Phys. Rev. Lett. 94 (2005) 166105.

[26] J. Müller, W. van Sarloos, Morphological instability and dynamics of fronts in bacterial growth models with nonlinear diffusion, Phys. Rev. E 65 (2002) 061111.

[27] E.D. Lozansky, O.B. Firsov, Theory of the initial stage of streamer propagation, J. Phys. D Appl. Phys. 6 (1973) 976.

[28] S.K. Dhali, P.F. Williams, Two-dimensional studies of streamers in gases, J. Appl. Phys. 62 (1987) 4696.

[29] P.A. Vitello, B.M. Penetrante, J.N. Bardsley, Simulation of negative-streamer dynamics in nitrogen, Phys. Rev. E 49 (1994) 5574.

[30] Yu.P. Raizer, Gas Discharge Physics, Springer, Berlin, 1991.

[31] E.M. Bazelyan, Yu.P. Raizer, Spark Discharge, CRC Press, Boca Raton, FL, 1998.

[32] U. Ebert, W. van Saarloos, C. Caroli, Propagation and structure of planar streamer fronts, Phys. Rev. E 55 (1997) 1530.

[33] U. Ebert, C. Montijn, T.M.P. Briels, W. Hundsdorfer, B. Meulenbroek, A. Rocco E.M. van Veldhuizen, The multiscale nature of streamers, Plasma Sources Sci. Technol. 15 (2006) S118. 
[34] F. Brau, A. Luque, B. Meulenbroek, U. Ebert, L. Schäfer, Construction and test of a moving boundary model for negative streamer discharges, Phys. Rev. E 77 (2008) 026219

[35] B. Meulenbroek, U. Ebert, L. Schäfer, Regularization of moving boundaries in a Laplacian field by a mixed Dirichlet-Neumann boundary condition: Exact results, Phys. Rev. Lett. 95 (2005) 195004.

[36] U. Ebert, B. Meulenbroek, L. Schäfer, Convective stabilization of a Laplacian moving boundary problem with kinetic undercooling, SIAM J. Appl. Math. 68 (2007) 292.

[37] S.J. Chapman, J.R. King, The selection of Saffman-Taylor fingers by kinetic undercooling, J. Eng. Math. 46 (2003) 1.

[38] A. Luque, F. Brau, U. Ebert, Saffman-Taylor streamers: Mutual finger interaction in spark formation, Phys. Rev. E 78 (2008) 016206.

[39] M. Arrayás, U. Ebert, W. Hundsdorfer, Spontaneous branching of anodedirected streamers between planar electrodes, Phys. Rev. Lett. 88 (2002) 174502.

[40] C. Montijn, U. Ebert, W. Hundsdorfer, Numerical convergence of the branching time of negative streamers, Phys. Rev. E 73 (2006) 065401
[41] M. Arrayás, U. Ebert, Stability of negative ionization fronts: regularization by electric screening? Phys. Rev. E 69 (2004) 056220.

[42] G. Derks, U. Ebert, B. Meulenbroek, Laplacian instability of planar streamer ionization fronts - An example of pulled front analysis, J. Nonlinear Sci. 18 (2008) 551.

[43] C.-Y. Kao, F. Brau, U. Ebert, L. Schäfer, S. Tanveer, A moving boundary problem motivated by electric breakdown: II. Initial value problem, Physica D (in preparation).

[44] P.Ya. Polubarinova-Kochina, On the motion of the oil contour, Dokl. Akad. Nauk. SSSR 47 (1945) 254 (in Russian).

[45] L.A. Galin, Unsteady filtration with a free surface, Dokl. Akad. Nauk. SSSR 47 (1945) 246 (in Russian).

[46] M. Günther, G. Prokert, On travelling wave solutions for a moving boundary problem of Hele-Shaw type, IMA J. Appl. Math. 74 (2009) 107.

[47] E.T. Whittaker, G.N. Watson, A course of modern analysis, fourth edition, Cambridge Univ., 1927.

[48] F. Olver, Asymptotics and Special Functions, AK Peters, Wellesley, MA, 1997 